$\underline{\text { Preprint typeset in JHEP style. - PAPER VERSION }}$

CPTh/S 635.0798

\title{
On power corrections in the dispersive approach
}

\author{
G. Grunberg* \\ Centre de Physique Théorique de l' Ecole Polytechnique (CNRS UMR C7644), \\ 91128 Palaiseau Cedex, France \\ E-mail: grunberg@cpht.polytechnique.fr
}

\begin{abstract}
Power corrections in QCD (both conventional and unconventional ones arising from the ultraviolet region) are discussed within the infrared finite coupling-dispersive approach. It is shown how power corrections in Minkowskian quantities can be derived from the corresponding ones in associated Euclidean quantities through analyticity, allowing a parametrization in term of the Euclidean coupling and a renormalon-free perturbative expansion. It is argued that one should in general expect coefficients functions computed in the true non-perturbative vacuum to differ from the standard perturbative ones, even without assuming new physics. A phenomenology of $1 / Q^{2}$ terms arising from eventual new physics of ultraviolet origin is also set-up. Models for non-perturbative contributions to the (universal) QCD coupling are suggested. Issues of renormalization scheme dependence are commented upon.
\end{abstract}

\footnotetext{
${ }^{*}$ Research supported in part by the EC program "Training and Mobility of Researchers", Network "QCD and Particle Structure", contract ERBFMRXCT980194.
} 


\section{Contents}

1. Introduction 1

2. Parametrization of infrared power corrections 2

3. Cancellation of IR renormalons $\quad 6$

4. The Euclidean-Minkowskian connection 9

5. Ultraviolet power corrections 13

6. Ansätze for the non-perturbative contributions to the universal $\begin{array}{ll}\text { QCD running coupling } & 21\end{array}$

6.1 QED inspired models 21

6.2 Models based on the "analytic perturbation theory" coupling 23

7. Scheme dependence issues $\quad 24$

8. Summary and Conclusions 26

A. Power corrections in Euclidean quantities 28

B. Power corrections in Minkowskian quantities $\quad 31$

C. Expressing Minkowskian power corrections in term of $\delta \bar{\alpha}_{s} \quad 36$

\section{Introduction}

The study of power corrections in QCD has been the subject of active investigations in recent years. Their importance for a precise determination of $\alpha_{s}$ has been recognized, and various techniques (renormalons, finite gluon mass, dispersive approach) have been devised to cope with situations where the standard operator product expansion (OPE) does not apply (for recent reviews see ref.[1, 2]). In this paper (which is a revised and extended version of [3]), I investigate various issues of the dispersive approach [4], based on the notion of an infrared (IR) regular [5, 6] universal QCD coupling $\bar{\alpha}_{s}$, putting emphasis on Minkowskian quantities. After a brief review of this method in section 2 , where the possibility of power corrections arising 
from non-perturbative contributions of $\bar{\alpha}_{s}$ to the ultraviolet (UV) region is pointed out, I first discuss the "standard" power corrections of infrared origin, which depend on the (non-perturbative) low energy behavior of $\bar{\alpha}_{s}$. It has been recently realized $[7,8]$ that a renormalon free perturbative expansion can be set up in the general case of Minkowskian quantities. I give an explicit check of this statement in section 3 , where the useful concept of "IR regularized characteristic function" is introduced; the case of a "causal" [9] perturbative coupling is also briefly discussed there. In section 4, I give a very simple derivation of power corrections to Minkowskian quantities, as well as of the renormalon-free perturbative expansion, by relating them through $Q^{2}$ analyticity to the corresponding terms (straightforward to derive) in the associated Euclidean quantities. Section 5 discusses "ultraviolet" power corrections. They may arise either from a $\mathcal{O}\left(1 / k^{4}\right)$ power suppressed term in the coupling of "standard" IR origin, or from more hypothetical (implying new physics) $\mathcal{O}\left(1 / k^{2}\right)$ terms, which generate $1 / Q^{2}$ corrections. I show that a simple phenomenology for the channel-dependence of the latter type of contributions can be set up [10]. It is also

pointed out that coefficient functions of higher dimensional operators computed in the non-perturbative vacuum may differ from the standard perturbative ones, even without assuming new physics. In section 6 , some models supporting the existence of power corrections, not necessarily of the $1 / k^{2}$ type, to the running coupling $\bar{\alpha}_{s}$ itself are presented. A major difference with [3] is that I do not argue anymore that $1 / Q^{2}$ terms arise naturally in the framework of [4] from considerations of Landau pole cancellation. Section 7 deals with the potentially important renormalization sheme $(\mathrm{RS})$ dependence issue, and indicates a possible solution based on the RS independence of the (BLM-like [11]) dressed skeleton expansion [12]. A summary and conclusions are given in section 8. More technical issues are relegated to three appendices. A method to derive power corrections to Euclidean quantities in the dressed single gluon exchange approximation is described in Appendix A. Power corrections to Minkowskian quantities are discussed in Appendix B. Finally, Appendix $\mathrm{C}$ shows how one can express power corrections to Minkowskian quantities directly in term of the Euclidean coupling $\bar{\alpha}_{s}$ itself.

\section{Parametrization of infrared power corrections}

Consider the contribution to an Euclidean (quark dominated) observable arising from dressed virtual single gluon exchange, which takes the generic form (after subtraction of the Born term):

$$
D\left(Q^{2}\right) \equiv D\left(\Lambda^{2} / Q^{2}\right)=\int_{0}^{\infty} \frac{d k^{2}}{k^{2}} \bar{\alpha}_{s}\left(\Lambda^{2} / k^{2}\right) \Phi_{D}\left(k^{2} / Q^{2}\right)
$$

where $\Phi_{D}$ is the "distribution function" [13]. The "physical" running coupling $\bar{\alpha}_{s}\left(k^{2}\right) \equiv$ $\bar{\alpha}_{s}\left(\Lambda^{2} / k^{2}\right)$ is assumed to be IR regular, and thus must differ from the perturbative 
coupling $\bar{\alpha}_{s}^{P T}\left(k^{2}\right)$ (defined by the Borel sum eq.(3.1)), which is assumed ${ }^{1}$ in most of the paper to have a Landau singularity, by a non-perturbative piece $\delta \bar{\alpha}_{s}\left(k^{2}\right)$ which cancells the singularity:

$$
\bar{\alpha}_{s}\left(k^{2}\right)=\bar{\alpha}_{s}^{P T}\left(k^{2}\right)+\delta \bar{\alpha}_{s}\left(k^{2}\right)
$$

$\bar{\alpha}_{s}\left(k^{2}\right)$ should be understood as a universal QCD coupling (not to be confused with e.g. the $\overline{M S}$ coupling: I use the overbar to identify this specific coupling), an analogue of the Gell-Mann - Low QED effective charge, hopefully defined through an extension to QCD of the QED "dressed skeleton expansion" $[11,12]$. Such a program, which would give a firm field theoretical basis to the "naive non-abelization" procedure $[14,15]$ familiar in renormalons calculations, has been initiated in [16]. The universal QCD coupling is presently known $[17,6,16]$ only as an expansion in the $\overline{M S}$ scheme up to next to leading order ${ }^{2}$. In the "large $\beta_{0}$ " limit of QCD, as implemented through the "naive non-abelization" procedure, $\bar{\alpha}_{s}\left(k^{2}\right)$ coincides with the V-scheme coupling [11] (but differs from it at finite $\beta_{0}$ ). Consequently:

$$
\begin{aligned}
D\left(Q^{2}\right) & =\int_{0}^{\infty} \frac{d k^{2}}{k^{2}} \bar{\alpha}_{s}^{P T}\left(k^{2}\right) \Phi_{D}\left(k^{2} / Q^{2}\right)+\int_{0}^{\infty} \frac{d k^{2}}{k^{2}} \delta \bar{\alpha}_{s}\left(k^{2}\right) \Phi_{D}\left(k^{2} / Q^{2}\right) \\
& \equiv D_{P T}\left(Q^{2}\right)+\delta D\left(Q^{2}\right)
\end{aligned}
$$

where $\delta D\left(Q^{2}\right)$ contains the power corrections. To determine the various types of power contributions, it is appropriate $[5,13]$ to disentangle long from short distances $[18,19,20]$ with an IR cut-off $\mu_{I}=\mathcal{O}(\Lambda)$ :

$$
\begin{aligned}
D\left(Q^{2}\right) & =\int_{0}^{\mu_{I}^{2}} \frac{d k^{2}}{k^{2}} \bar{\alpha}_{s}\left(k^{2}\right) \Phi_{D}\left(k^{2} / Q^{2}\right)+\int_{\mu_{I}^{2}}^{\infty} \frac{d k^{2}}{k^{2}} \bar{\alpha}_{s}\left(k^{2}\right) \Phi_{D}\left(k^{2} / Q^{2}\right) \\
& \equiv D_{I R}\left(Q^{2}\right)+D_{U V}\left(Q^{2}\right)
\end{aligned}
$$

It is convenient to further disentangle perturbative from non-perturbative contributions in the short distance part, and set:

$$
\begin{aligned}
D_{U V}\left(Q^{2}\right) & =\int_{\mu_{I}^{2}}^{\infty} \frac{d k^{2}}{k^{2}} \bar{\alpha}_{s}^{P T}\left(k^{2}\right) \Phi_{D}\left(k^{2} / Q^{2}\right)+\int_{\mu_{I}^{2}}^{\infty} \frac{d k^{2}}{k^{2}} \delta \bar{\alpha}_{s}\left(k^{2}\right) \Phi_{D}\left(k^{2} / Q^{2}\right) \\
& \equiv D_{U V}^{P T}\left(Q^{2}\right)+\delta D_{U V}\left(Q^{2}\right)
\end{aligned}
$$

Thus:

$$
D\left(Q^{2}\right)=D_{I R}\left(Q^{2}\right)+D_{U V}^{P T}\left(Q^{2}\right)+\delta D_{U V}\left(Q^{2}\right)
$$

$D_{I R}\left(Q^{2}\right)$ yields, for large $Q^{2}$, "long distance " power contributions which correspond to the standard OPE "condensates". If the Feynman diagram kernel $\Phi_{D}\left(k^{2} / Q^{2}\right)$ is

\footnotetext{
${ }^{1}$ See however the "causal" perturbative coupling case in section 3 and the remarks in section 5 .

${ }^{2}$ The constant term in the next to leading order expansion of $\bar{\alpha}_{s}$ in the $\overline{M S}$ scheme actually differ by a factor of $\pi^{2}$ in [17] and [16]. This discrepancy should be clarified.
} 
$\mathcal{O}\left[\left(k^{2} / Q^{2}\right)^{n}\right]$ at small $k^{2}$, this piece contributes an $\mathcal{O}\left[\left(\Lambda^{2} / Q^{2}\right)^{n}\right]$ term from a dimension $n$ condensate, with the normalization given by a low energy average of the IR regular coupling $\bar{\alpha}_{s}$ (see Appendix A). $D_{U V}^{P T}\left(Q^{2}\right)$ represents a form of "regularized perturbation theory" (choosing the IR cut-off $\mu_{I}$ above the Landau pole), where the long distance part of the perturbative contribution has been removed:

$$
\begin{aligned}
D_{U V}^{P T}\left(Q^{2}\right) & =\int_{0}^{\infty} \frac{d k^{2}}{k^{2}} \bar{\alpha}_{s}^{P T}\left(k^{2}\right) \Phi_{D}\left(k^{2} / Q^{2}\right)-\int_{0}^{\mu_{I}^{2}} \frac{d k^{2}}{k^{2}} \bar{\alpha}_{s}^{P T}\left(k^{2}\right) \Phi_{D}\left(k^{2} / Q^{2}\right) \\
& \equiv D_{P T}\left(Q^{2}\right)-D_{I R}^{P T}\left(Q^{2}\right)
\end{aligned}
$$

The last term $\delta D_{U V}\left(Q^{2}\right)$ in eq.(2.6) yields, unless $\delta \bar{\alpha}_{s}\left(k^{2}\right)$ is highly suppressed, additional "ultraviolet" power contributions at large $Q^{2}$. They are usually neglected, but I shall return to them in section 5 . Note that we have:

$$
\delta D\left(Q^{2}\right)=\delta D_{I R}\left(Q^{2}\right)+\delta D_{U V}\left(Q^{2}\right)
$$

with:

$$
\delta D_{I R}\left(Q^{2}\right) \equiv \int_{0}^{\mu_{I}^{2}} \frac{d k^{2}}{k^{2}} \delta \bar{\alpha}_{s}\left(k^{2}\right) \Phi_{D}\left(k^{2} / Q^{2}\right)=D_{I R}\left(Q^{2}\right)-D_{I R}^{P T}\left(Q^{2}\right)
$$

A derivation of the power corrections in $\delta D\left(Q^{2}\right)$ can be found in Appendix A.

One would like to give a parametrization of IR power corrections for Minkowskian quantities analoguous to eq.(2.6), i.e. in term of $\bar{\alpha}_{s}$ itself. The problem is that a representation such as eq.(2.1) in general does not exist [13]. For a sufficiently inclusive Minkowskian quantity $R$, we have instead a representation in term of the time like discontinuity of the coupling $[15,4]$ :

$$
\begin{aligned}
R\left(Q^{2}\right) & =\int_{0}^{\infty} \frac{d \mu^{2}}{\mu^{2}} \bar{\rho}_{s}\left(\mu^{2}\right)\left[\mathcal{F}_{R}\left(\mu^{2} / Q^{2}\right)-\mathcal{F}_{R}(0)\right] \\
& \equiv \int_{0}^{\infty} \frac{d \mu^{2}}{\mu^{2}} \bar{\alpha}_{e f f}\left(\mu^{2}\right) \dot{\mathcal{F}}_{R}\left(\mu^{2} / Q^{2}\right)
\end{aligned}
$$

where:

$$
\bar{\rho}_{s}\left(\mu^{2}\right)=-\frac{1}{2 \pi i} \operatorname{Disc}\left\{\bar{\alpha}_{s}\left(-\mu^{2}\right)\right\} \equiv-\frac{1}{2 \pi i}\left\{\bar{\alpha}_{s}\left[-\left(\mu^{2}+i \epsilon\right)\right]-\bar{\alpha}_{s}\left[-\left(\mu^{2}-i \epsilon\right)\right]\right\}
$$

is the time like "spectral density", and the "effective coupling" $\bar{\alpha}_{e f f}\left(\mu^{2}\right)$ is defined by:

$$
\frac{d \bar{\alpha}_{e f f}}{d \ln \mu^{2}}=\bar{\rho}_{s}\left(\mu^{2}\right)
$$

$\bar{\alpha}_{s}$ is assumed to satisfy the dispersion relation :

$$
\begin{aligned}
\bar{\alpha}_{s}\left(k^{2}\right) & =-\int_{0}^{\infty} \frac{d \mu^{2}}{\mu^{2}+k^{2}} \bar{\rho}_{s}\left(\mu^{2}\right) \\
& \equiv k^{2} \int_{0}^{\infty} \frac{d \mu^{2}}{\left(\mu^{2}+k^{2}\right)^{2}} \bar{\alpha}_{e f f}\left(\mu^{2}\right)
\end{aligned}
$$


which implies in particular the absence of Landau singularity.

The "characteristic function" $\mathcal{F}_{R}$ in eq.(2.10) is computed from the one-loop Feynman diagrams with a finite gluon mass $\mu$, and $\dot{\mathcal{F}}_{R} \equiv-d \mathcal{F}_{R} / d \ln \mu^{2}$. It is usually composed of two distinct pieces, for instance:

$$
\mathcal{F}_{R}\left(\mu^{2} / Q^{2}\right)=\left\{\begin{array}{cc}
\mathcal{F}_{R,(-)}\left(\mu^{2} / Q^{2}\right) & \left(0<\mu^{2}<Q^{2}\right) \\
\mathcal{F}_{R,(+)}\left(\mu^{2} / Q^{2}\right) & \left(\mu^{2}>Q^{2}\right)
\end{array}\right.
$$

where $\mathcal{F}_{R,(-)}$ is the sum of a real and a virtual contribution, while $\mathcal{F}_{R,(+)}$ contains only the virtual contribution, and may vanish identically, as in the case of thrust. This feature prevents (see the comment below eq.(3.14)) a representation of $R$ similar to eq.(2.1) to be reconstructed from eq.(2.10) using analyticity. Nevertheless, as pointed out in $[7,8]$, it is still possible to parametrize the IR power corrections in term of $\bar{\alpha}_{s}$ (a parametrization of power corrections in term of quantities related to the "Minkowskian coupling" $\bar{\alpha}_{e f f}$ is possible, but cumbersome (see Appendix B)).

The first observation [3] is that the power correction piece $\delta R\left(Q^{2}\right)$ in:

$$
R\left(Q^{2}\right)=R_{P T}\left(Q^{2}\right)+\delta R\left(Q^{2}\right)
$$

(where $R_{P T}\left(Q^{2}\right)$ is defined to be the Borel sum, see eq.(3.4) below) can be expressed as an integral over the non-perturbative modification $\delta \bar{\alpha}_{s}$ of the coupling in eq. (2.2). Provided $\delta \bar{\alpha}_{s}\left(k^{2}\right)$ decreases sufficiently fast at large $k^{2}$, one can indeed show (Appendix C) that:

$$
\delta R\left(Q^{2}\right) \simeq \int_{0}^{\infty} \frac{d k^{2}}{k^{2}} \delta \bar{\alpha}_{s}\left(k^{2}\right) \Phi_{R}\left(k^{2} / Q^{2}\right)
$$

where $\Phi_{R}\left(k^{2} / Q^{2}\right)$ is the discontinuity at $\mu^{2}=-k^{2}<0$ of the "low gluon mass" piece $\mathcal{F}_{R,(-)}$ of the characteristic function. The proof of eq.(2.16) is actually not straightforward, if one assumes the perturbative coupling $\bar{\alpha}_{s}^{P T}$ (hence also $\delta \bar{\alpha}_{s}$ ) has a Landau singularity. The reason is that, if $\bar{\alpha}_{s}^{P T}$ is not "causal", i.e. does not satisfy eq.(2.13) with $\bar{\alpha}_{e f f} \rightarrow \bar{\alpha}_{e f f}^{P T}, \delta \bar{\alpha}_{s}$ is not related to its time-like analogue $\delta \bar{\alpha}_{e f f}$ (where $\left.\bar{\alpha}_{e f f}=\bar{\alpha}_{e f f}^{P T}+\delta \bar{\alpha}_{e f f}\right)$ by the dispersion relation eq.(2.13). Moreover, $R_{P T}$ and $\delta R$ are not given (see Appendix B) by eq.(2.10) with $\bar{\alpha}_{e f f}$ substituted respectively by $\bar{\alpha}_{e f f}^{P T}$ and $\delta \bar{\alpha}_{e f f}$.

In a second step, one introduces an IR cutoff $\mu_{I}$ into eq.(2.16) and substitute $\delta \bar{\alpha}_{s}\left(k^{2}\right)=\bar{\alpha}_{s}\left(k^{2}\right)-\bar{\alpha}_{s}^{P T}\left(k^{2}\right)$ in the low energy piece. Thus, neglecting the high energy integral above $\mu_{I}^{2}$, one gets:

$$
\delta R\left(Q^{2}\right) \simeq \delta R_{I R}\left(Q^{2}\right)
$$

with:

$$
\delta R_{I R}\left(Q^{2}\right) \equiv \int_{0}^{\mu_{I}^{2}} \frac{d k^{2}}{k^{2}} \delta \bar{\alpha}_{s}\left(k^{2}\right) \Phi_{R}\left(k^{2} / Q^{2}\right)=R_{I R}\left(Q^{2}\right)-R_{I R}^{P T}\left(Q^{2}\right)
$$


where:

$$
R_{I R}\left(Q^{2}\right) \equiv \int_{0}^{\mu_{I}^{2}} \frac{d k^{2}}{k^{2}} \bar{\alpha}_{s}\left(k^{2}\right) \Phi_{R}\left(k^{2} / Q^{2}\right)
$$

and:

$$
R_{I R}^{P T}\left(Q^{2}\right) \equiv \int_{0}^{\mu_{I}^{2}} \frac{d k^{2}}{k^{2}} \bar{\alpha}_{s}^{P T}\left(k^{2}\right) \Phi_{R}\left(k^{2} / Q^{2}\right)
$$

One deduces $[7,8]$ :

$$
\begin{aligned}
R\left(Q^{2}\right) & \simeq R_{P T}\left(Q^{2}\right)+\delta R_{I R}\left(Q^{2}\right) \\
& =R_{I R}\left(Q^{2}\right)+R_{U V}^{P T}\left(Q^{2}\right)
\end{aligned}
$$

with:

$$
R_{U V}^{P T}\left(Q^{2}\right) \equiv R_{P T}\left(Q^{2}\right)-R_{I R}^{P T}\left(Q^{2}\right)
$$

Eq. (2.21), which is correct only if $\delta \bar{\alpha}_{s}\left(k^{2}\right)$ decreases fast enough at large $k^{2}$, is the analogue of eq.(2.6) (with the last "UV" piece neglected), and the "infrared" power corrections are again parametrized in term of a low energy average of $\bar{\alpha}_{s}$. The only difference is that the "regularized perturbation theory" piece $R_{U V}^{P T}$ (just as $R_{P T}$ ) can no more be written as an integral, cut-off in the infrared, over the perturbative part of the coupling, although it is still a renormalon free quantity.

\section{Cancellation of IR renormalons}

To check the latter property explicitly, it is convenient to revert to the original representation eq.(2.10), and express the Borel transform of $R_{U V}^{P T}$ in term of that of $\bar{\alpha}_{e f f}^{P T}$. One introduces the (RS invariant $\left.[21,22,23]\right)$ Borel representations ${ }^{3}$ of $\bar{\alpha}_{s}^{P T}\left(k^{2}\right)$ and $\bar{\alpha}_{e f f}^{P T}\left(\mu^{2}\right)$ :

$$
\bar{\alpha}_{s}^{P T}\left(k^{2}\right)=\int_{0}^{\infty} d z \exp \left(-z \beta_{0} \ln \frac{k^{2}}{\Lambda^{2}}\right) \quad \tilde{\alpha}_{s}(z)
$$

and:

$$
\bar{\alpha}_{e f f}^{P T}\left(\mu^{2}\right)=\int_{0}^{\infty} d z \exp \left(-z \beta_{0} \ln \frac{\mu^{2}}{\Lambda^{2}}\right) \tilde{\alpha}_{e f f}(z)
$$

( $\beta_{0}$ is (minus) the one loop beta function coefficient) where the (RS invariant) Borel transforms $\tilde{\alpha}_{e f f}(z)$ and $\tilde{\alpha}_{s}(z)$ are related by $[3,23]$ :

$$
\tilde{\alpha}_{e f f}(z)=\frac{\sin \left(\pi \beta_{0} z\right)}{\pi \beta_{0} z} \tilde{\alpha}_{s}(z)
$$

Substituting $\bar{\alpha}_{e f f}\left(\mu^{2}\right)$ in eq.(2.10) with $\bar{\alpha}_{e f f}^{P T}\left(\mu^{2}\right)$ as given by eq.(3.2), one gets the (RS invariant) Borel representation:

$$
R_{P T}\left(Q^{2}\right)=\int_{0}^{\infty} d z \tilde{\alpha}_{e f f}(z)\left[\int_{0}^{\infty} \frac{d \mu^{2}}{\mu^{2}} \dot{\mathcal{F}}_{R}\left(\mu^{2} / Q^{2}\right) \exp \left(-z \beta_{0} \ln \frac{\mu^{2}}{\Lambda^{2}}\right)\right]
$$

\footnotetext{
${ }^{3}$ An exact expression for $\tilde{\alpha}_{s}(z)$ in the case $\bar{\alpha}_{s}^{P T}\left(k^{2}\right)$ satisfies the two loop renormalization group equation is given in [21].
} 
Note that, since the representation eq.(3.2) is valid only for $\mu^{2}>\Lambda^{2}$, and $\bar{\alpha}_{e f f}^{P T}\left(\mu^{2}\right)$ has a non-trivial IR fixed point [15], the Borel sum $R_{P T}\left(Q^{2}\right)$ is different [25, 26], as mentionned below eq.(2.16), from the corresponding "gluon mass" integral [15]:

$$
R_{A P T}\left(Q^{2}\right) \equiv \int_{0}^{\infty} \frac{d \mu^{2}}{\mu^{2}} \bar{\alpha}_{e f f}^{P T}\left(\mu^{2}\right) \dot{\mathcal{F}}_{R}\left(\mu^{2} / Q^{2}\right)
$$

Next consider $R_{I R}\left(Q^{2}\right)$ (eq.(2.19)). Using the dispersion relation eq.(2.13), one gets the "Minkowskian" representation (analogue of eq.(2.10)):

$$
R_{I R}\left(Q^{2}\right)=\int_{0}^{\infty} \frac{d \mu^{2}}{\mu^{2}} \bar{\alpha}_{e f f}\left(\mu^{2}\right) \dot{\mathcal{F}}_{R, I R}\left(\mu^{2}, Q^{2}\right)
$$

with:

$$
\dot{\mathcal{F}}_{R, I R}\left(\mu^{2}, Q^{2}\right) \equiv \mu^{2} \int_{0}^{\mu_{I}^{2}} \frac{d k^{2}}{\left(k^{2}+\mu^{2}\right)^{2}} \Phi_{R}\left(k^{2} / Q^{2}\right)
$$

and $\dot{\mathcal{F}}_{R, I R}\left(\mu^{2}, Q^{2}\right)$ is written as a function of two variables to emphasize that a third scale $\left(\mu_{I}\right)$ is involved. Substituting $\bar{\alpha}_{e f f}$ in eq.(3.6) with eq.(3.2), one finds similarly the Borel representation of $R_{I R}^{P T}\left(Q^{2}\right)$ :

$$
R_{I R}^{P T}\left(Q^{2}\right)=\int_{0}^{\infty} d z \tilde{\alpha}_{e f f}(z)\left[\int_{0}^{\infty} \frac{d \mu^{2}}{\mu^{2}} \dot{\mathcal{F}}_{R, I R}\left(\mu^{2}, Q^{2}\right) \exp \left(-z \beta_{0} \ln \frac{\mu^{2}}{\Lambda^{2}}\right)\right]
$$

(using the original definition of $R_{I R}^{P T}$ in eq.(2.20), one could have also written the Borel transform in term of $\Phi_{R}$, but this is less useful for Minkowskian quantities). One deduces from eq.(2.22):

$$
R_{U V}^{P T}\left(Q^{2}\right)=\int_{0}^{\infty} d z \tilde{\alpha}_{e f f}(z)\left[\int_{0}^{\infty} \frac{d \mu^{2}}{\mu^{2}} \dot{\mathcal{F}}_{R, U V}\left(\mu^{2}, Q^{2}\right) \exp \left(-z \beta_{0} \ln \frac{\mu^{2}}{\Lambda^{2}}\right)\right]
$$

where:

$$
\dot{\mathcal{F}}_{R, U V}\left(\mu^{2}, Q^{2}\right) \equiv \dot{\mathcal{F}}_{R}\left(\mu^{2} / Q^{2}\right)-\dot{\mathcal{F}}_{R, I R}\left(\mu^{2}, Q^{2}\right)
$$

is the "IR regularized" characteristic function. Again, the Borel sums $R_{I R}^{P T}$ and $R_{U V}^{P T}$ are different from the corresponding "gluon mass" integrals

$$
R_{I R}^{A P T}\left(Q^{2}\right) \equiv \int_{0}^{\infty} \frac{d \mu^{2}}{\mu^{2}} \bar{\alpha}_{e f f}^{P T}\left(\mu^{2}\right) \dot{\mathcal{F}}_{R, I R}\left(\mu^{2}, Q^{2}\right)
$$

and

$$
R_{U V}^{A P T}\left(Q^{2}\right) \equiv \int_{0}^{\infty} \frac{d \mu^{2}}{\mu^{2}} \bar{\alpha}_{e f f}^{P T}\left(\mu^{2}\right) \dot{\mathcal{F}}_{R, U V}\left(\mu^{2}, Q^{2}\right)
$$

The effect of the subtracted term in eq.(3.10) is to remove any potential non-analytic term in the small $\mu^{2}$ expansion of $\dot{\mathcal{F}}_{R, U V}\left(\mu^{2}, Q^{2}\right)$ (now distinct from its large $Q^{2}$ behavior, since there is a third scale $\mu_{I}$ ), by introducing an IR cutoff $\mu_{I}$ in the dispersive integral over $\Phi_{R}$ (see eq.(C.2) or (C.6), and also eq.(3.16) below). It 
follows that the renormalons singularities in eq.(3.9) can only be simple poles, which are however cancelled by the zeroes of the sin factor in eq.(3.3) $\left(\tilde{\alpha}_{s}(z)\right.$ itself is assumed throughout to have no renormalons). The Borel sum eq.(3.9) is thus renormalon-free and unambiguous, as expected.

A similar formalism also applies $[15,4]$ to Euclidean quantities. Indeed, proceeding as for eq.(3.6) and using the dispersion relation eq.(2.13) into the "Euclidean" representation eq.(2.1) gives the "Minkowskian" representation:

$$
D\left(Q^{2}\right)=\int_{0}^{\infty} \frac{d \mu^{2}}{\mu^{2}} \bar{\alpha}_{e f f}\left(\mu^{2}\right) \dot{\mathcal{F}}_{D}\left(\mu^{2} / Q^{2}\right)
$$

where the Euclidean characteristic function $\mathcal{F}_{D}$ is related to the corresponding distribution function $\Phi_{D}$ by the dispersion relation $[27,13]$ :

$$
\dot{\mathcal{F}}_{D}\left(\mu^{2} / Q^{2}\right)=\mu^{2} \int_{0}^{\infty} \frac{d k^{2}}{\left(k^{2}+\mu^{2}\right)^{2}} \Phi_{D}\left(k^{2} / Q^{2}\right)
$$

Note that $\mathcal{F}_{D}$, at the difference of $\mathcal{F}_{R}$, must be made of a single piece, which shows that an Euclidean representation is indeed not possible for $R$. We then have:

$$
D_{U V}^{P T}\left(Q^{2}\right)=\int_{0}^{\infty} d z \tilde{\alpha}_{e f f}(z)\left[\int_{0}^{\infty} \frac{d \mu^{2}}{\mu^{2}} \dot{\mathcal{F}}_{D, U V}\left(\mu^{2}, Q^{2}\right) \exp \left(-z \beta_{0} \ln \frac{\mu^{2}}{\Lambda^{2}}\right)\right]
$$

with:

$$
\dot{\mathcal{F}}_{D, U V}\left(\mu^{2}, Q^{2}\right) \equiv \mu^{2} \int_{\mu_{I}^{2}}^{\infty} \frac{d k^{2}}{\left(k^{2}+\mu^{2}\right)^{2}} \Phi_{D}\left(k^{2} / Q^{2}\right)
$$

where the IR cut-off is explicit. It is also clear that the small $\mu^{2}$ behavior of $\dot{\mathcal{F}}_{D, U V}\left(\mu^{2}, Q^{2}\right)$ is analytic. The representation eq.(3.15) shall be used in section 4 . Case of a "causal" perturbative coupling: one can show that eq.(2.21) is still valid, and is in fact exact ${ }^{4}$ (with $R_{U V}^{P T}\left(Q^{2}\right)$ as in eq.(3.9)), if $\delta \bar{\alpha}_{s}\left(k^{2}\right) \equiv 0$. Then $\bar{\alpha}_{s}^{P T}$ is "causal" and satisfies by itself the dispersion relation eq.(2.13). This is known to occur in QCD for a large enough number of flavors, where the perturbative coupling has a non-trivial IR fixed point and no (real or complex) Landau singularity [9]. In such a case, $\bar{\alpha}_{s}^{P T}=\bar{\alpha}_{s}^{A P T}$, where $\bar{\alpha}_{s}^{A P T}$ is the "analytic" perturbation theory coupling $[24,3,9,15]$ of eq.(B.3) and (6.11), and we have:

$$
R\left(Q^{2}\right)=R_{A P T}\left(Q^{2}\right)=R_{I R}^{A P T}\left(Q^{2}\right)+R_{U V}^{A P T}\left(Q^{2}\right)
$$

where $R_{A P T}, R_{I R}^{A P T}$ and $R_{U V}^{A P T}$ are the "gluon mass" integrals of eq.(3.5), (3.11) and (3.12). But, using eq.(B.3), we get:

$$
R_{I R}^{A P T}\left(Q^{2}\right)=\int_{0}^{\mu_{I}^{2}} \frac{d k^{2}}{k^{2}} \bar{\alpha}_{s}^{A P T}\left(k^{2}\right) \Phi_{R}\left(k^{2} / Q^{2}\right)=\int_{0}^{\mu_{I}^{2}} \frac{d k^{2}}{k^{2}} \bar{\alpha}_{s}^{P T}\left(k^{2}\right) \Phi_{R}\left(k^{2} / Q^{2}\right)
$$

\footnotetext{
${ }^{4}$ For Euclidean quantities, this statement follows from the results in $[25,26]$.
} 
where in the second step I used that $\bar{\alpha}_{s}^{P T}$ is causal. On the other hand one can show, even if $\bar{\alpha}_{s}^{P T}$ is not causal (see Appendix B) that power corrections in $R_{U V}^{A P T}$ involve only analytic moments of $\bar{\alpha}_{e f f}^{P T}$ (the $b_{n}^{A P T}$ 's of eq.(B.21)), as a consequence of the analytic behavior of $\dot{\mathcal{F}}_{R, U V}\left(\mu^{2}, Q^{2}\right)$ at small $\mu^{2}$ (and despite the presence of non-analytic terms in the large $Q^{2}$ behavior). These moments turn out to vanish if $\bar{\alpha}_{s}^{P T}$ is causal. Thus for a causal coupling $R_{U V}^{A P T}\left(Q^{2}\right)$ (eq.(3.12)) coincides with the Borel sum $R_{U V}^{P T}\left(Q^{2}\right)$ (eq.(3.9)), and consequently:

$$
R_{A P T}\left(Q^{2}\right)=R_{I R}^{A P T}\left(Q^{2}\right)+R_{U V}^{P T}\left(Q^{2}\right)
$$

which is just eq.(2.21) in this case. One can similarly show, if the perturbative coupling is "causal", that analytic terms in the small $\mu^{2}$ behavior of $\dot{\mathcal{F}}_{R}\left(\mu^{2} / Q^{2}\right)$ can contribute no power corrections, and therefore $R_{A P T}\left(Q^{2}\right)$ differs from the Borel sum $R_{P T}\left(Q^{2}\right)$ only by the "OPE-compatible" power corrections arising from the nonanalytic terms. Note also $R_{I R}^{P T}\left(Q^{2}\right)$ is no more identical, due to the non-trivial IR fixed point of $\bar{\alpha}_{s}^{P T}$, to the right hand side of eq.(3.18), and should rather be replaced in eq.(2.20) and (2.22) by the corresponding Borel sum eq.(3.8).

\section{The Euclidean-Minkowskian connection}

The regularization procedure described in the previous section is rather formal, and provides no physical picture of $\dot{\mathcal{F}}_{R, U V}$ as an IR cutoff Feynman diagram (as opposed to $\left.\dot{\mathcal{F}}_{D, U V}\right)$. A more transparent interpretation can be given if the Minkowskian quantity $R$ is related to the time-like discontinuity of an Euclidean quantity $D$, i.e. I shall assume $D$ satisfies the dispersion relation:

$$
D\left(Q^{2}\right)=Q^{2} \int_{0}^{\infty} \frac{d Q^{\prime 2}}{\left(Q^{\prime 2}+Q^{2}\right)^{2}} R\left(Q^{\prime 2}\right)
$$

which implies (if $R\left(Q^{2}\right)$ vanishes at $Q^{2}=0$ ) the inverse relation:

$$
R\left(Q^{2}\right)=\frac{1}{2 \pi i} \oint_{\left|Q^{\prime 2}\right|=Q^{2}} \frac{d Q^{\prime 2}}{Q^{\prime 2}} D\left(Q^{\prime 2}\right)
$$

The main point of this section is that each term in eq.(2.21) can be obtained from the corresponding ones in eq.(2.6) through the relation eq.(4.2) (for large enough $Q^{2}$ ), i.e. by taking their (integrated) time-like discontinuity, after analytic continuation to complex $Q^{2}$ (the latter formulation being valid for all $Q^{2}$ ). Note that these statements do not imply that these terms are necessarily related by the dispersion relation eq.(4.1). 
I first observe that eq.(3.13) and (3.14) allow an analytic continuation ${ }^{5}$ of $D\left(Q^{2}\right)$ to complex $Q^{2}$ (through continuation of the integrand, keeping $\mu^{2}$ (and $\Lambda^{2}$ ) real), and imply that $D\left(Q^{2}\right)$ does satisfy the dispersion relation eq.(4.1). Indeed, performing the change of variable: $k^{2}=\frac{\mu^{2} Q^{2}}{Q^{\prime 2}}$, eq.(3.14) can also be written as:

$$
\dot{\mathcal{F}}_{D}\left(\mu^{2} / Q^{2}\right)=Q^{2} \int_{0}^{\infty} \frac{d Q^{\prime 2}}{\left(Q^{\prime 2}+Q^{2}\right)^{2}} \Phi_{D}\left(\mu^{2} / Q^{\prime 2}\right)
$$

Inserting eq.(4.3) into eq.(3.13) reproduces eq.(4.1), with:

$$
R\left(Q^{2}\right)=\int_{0}^{\infty} \frac{d \mu^{2}}{\mu^{2}} \bar{\alpha}_{e f f}\left(\mu^{2}\right) \Phi_{D}\left(\mu^{2} / Q^{2}\right)
$$

As a byproduct, we also learn that [13]:

$$
\Phi_{D}\left(\mu^{2} / Q^{2}\right) \equiv \dot{\mathcal{F}}_{R}\left(\mu^{2} / Q^{2}\right)
$$

Furthermore, the inverse relation eq.(4.2) is also satisfied, since $R\left(Q^{2}\right)$ vanishes for $Q^{2} \rightarrow \infty$ (see previous footnote). Alternatively, I note that eq.(3.14) implies, since $\Phi_{D}\left(\mu^{2} / Q^{2}\right)$ vanishes at $\mu^{2}=0$ :

$$
\Phi_{D}\left(\mu^{2} / Q^{2}\right)=\frac{1}{2 \pi i} \oint_{\left|k^{2}\right|=\mu^{2}} \frac{d k^{2}}{k^{2}} \dot{\mathcal{F}}_{D}\left(k^{2} / Q^{2}\right)
$$

which can be written with the previous change of variable:

$$
\dot{\mathcal{F}}_{R}\left(\mu^{2} / Q^{2}\right)=\frac{1}{2 \pi i} \oint_{\left|Q^{\prime 2}\right|=Q^{2}} \frac{d Q^{\prime 2}}{Q^{\prime 2}} \dot{\mathcal{F}}_{D}\left(\mu^{2} / Q^{\prime 2}\right)
$$

From eq.(3.13) and (4.7) one then deduces:

$$
\begin{aligned}
\frac{1}{2 \pi i} \oint_{\left|Q^{\prime 2}\right|=Q^{2}} \frac{d Q^{\prime 2}}{Q^{\prime 2}} D\left(Q^{\prime 2}\right) & =\int_{0}^{\infty} \frac{d \mu^{2}}{\mu^{2}} \bar{\alpha}_{e f f}\left(\mu^{2}\right) \frac{1}{2 \pi i} \oint_{\left|Q^{\prime 2}\right|=Q^{2}} \frac{d Q^{\prime 2}}{Q^{\prime 2}} \dot{\mathcal{F}}_{D}\left(\mu^{2} / Q^{\prime 2}\right) \\
& =\int_{0}^{\infty} \frac{d \mu^{2}}{\mu^{2}} \bar{\alpha}_{e f f}\left(\mu^{2}\right) \dot{\mathcal{F}}_{R}\left(\mu^{2} / Q^{2}\right)=R\left(Q^{2}\right)
\end{aligned}
$$

which proves eq.(4.2).

Consider next the term $D_{I R}\left(Q^{2}\right)$ in eq.(2.6), which contains the "IR" power corrections. For $\mu_{I}^{2}<Q^{2}$, one can identify $\Phi_{D}\left(k^{2} / Q^{2}\right)$ with the "low gluon mass piece" of $\dot{\mathcal{F}}_{R}\left(k^{2} / Q^{2}\right)$, and $D_{I R}\left(Q^{2}\right)$ (eq.(2.4)) may be analytically continued to complex

\footnotetext{
${ }^{5}$ Alternatively, one could continue $D\left(Q^{2}\right)$ to complex $\Lambda^{2}$ using eq.(2.1), keeping $k^{2}$ and $Q^{2}$ real (this is a simplified version of the method of [13]). Similarly, considering the analytic properties of $D\left(Q^{2}\right)$ in eq.(4.1) with respect to $\Lambda^{2}$, i.e. essentially with respect to the variable $1 / Q^{2}$, one can derive eq.(4.2) from the alternative assumption (which involves no non-perturbative physics) that $R\left(Q^{2}\right)$ vanishes for $Q^{2} \rightarrow \infty$.
} 
$Q^{2}$ through the continuation of the integrand, keeping $k^{2}$ (and $\Lambda^{2}$ ) real. Thus, for $Q^{2}>\mu_{I}^{2}$ :

$$
\frac{1}{2 \pi i} \oint_{\left|Q^{\prime 2}\right|=Q^{2}} \frac{d Q^{\prime 2}}{Q^{\prime 2}} D_{I R}\left(Q^{\prime 2}\right)=\int_{0}^{\mu_{I}^{2}} \frac{d k^{2}}{k^{2}} \bar{\alpha}_{s}\left(k^{2}\right) \frac{1}{2 \pi i} \oint_{\left|Q^{\prime 2}\right|=Q^{2}} \frac{d Q^{\prime 2}}{Q^{\prime 2}} \Phi_{D}\left(k^{2} / Q^{\prime 2}\right)
$$

Now, performing the change of variable $Q^{\prime 2}=\frac{k^{2} Q^{2}}{k^{\prime 2}}$, we get:

$$
\frac{1}{2 \pi i} \oint_{\left|Q^{\prime 2}\right|=Q^{2}} \frac{d Q^{\prime 2}}{Q^{\prime 2}} \Phi_{D}\left(k^{2} / Q^{\prime 2}\right)=\frac{1}{2 \pi i} \oint_{\left|k^{\prime 2}\right|=k^{2}} \frac{d k^{\prime 2}}{k^{\prime 2}} \dot{\mathcal{F}}_{R}\left(k^{\prime 2} / Q^{2}\right)
$$

where I also used eq.(4.5). Furthermore, assuming the absence of complex singularities in $\dot{\mathcal{F}}_{R}\left(k^{2} / Q^{2}\right)$ for small enough "gluon mass" $\left|k^{2}\right|$ (which is certainly the case if the low $k^{2}$ expansion of $\dot{\mathcal{F}}_{R}\left(k^{2} / Q^{2}\right)$ involves only elementary functions), Cauchy theorem yields for small enough $k^{2}\left(\Phi_{R}\right.$ vanishes at $\left.k^{2}=0\right)$ :

$$
\frac{1}{2 \pi i} \oint_{\left|k^{\prime 2}\right|=k^{2}} \frac{d k^{\prime 2}}{k^{\prime 2}} \dot{\mathcal{F}}_{R}\left(k^{\prime 2} / Q^{2}\right)=\Phi_{R}\left(k^{2} / Q^{2}\right)
$$

Eq.(4.11) is the analogue of eq.(4.6); being a finite "gluon mass" sum rule, it does not depend on the high $k^{2}$ behavior of $\dot{\mathcal{F}}_{R}$, and in particular still holds in presence of subtractions. We thus obtain, for large enough $Q^{2}$ :

$$
\Phi_{R}\left(k^{2} / Q^{2}\right)=\frac{1}{2 \pi i} \oint_{\left|Q^{\prime 2}\right|=Q^{2}} \frac{d Q^{\prime 2}}{Q^{\prime 2}} \Phi_{D}\left(k^{2} / Q^{\prime 2}\right)
$$

which shows (see eq.(2.19)) that we have:

$$
R_{I R}\left(Q^{2}\right)=\frac{1}{2 \pi i} \oint_{\left|Q^{\prime 2}\right|=Q^{2}} \frac{d Q^{\prime 2}}{Q^{\prime 2}} D_{I R}\left(Q^{\prime 2}\right)
$$

Note that if $\Phi_{D}$ were made of a single piece at all scales, one could apply the same argument to the whole $D\left(Q^{2}\right)$ to get an expression for $R$ in term of $\bar{\alpha}_{s}$.

Moreover, one can also show that:

$$
R_{U V}^{P T}\left(Q^{2}\right)=\frac{1}{2 \pi i} \oint_{\left|Q^{\prime 2}\right|=Q^{2}} \frac{d Q^{\prime 2}}{Q^{\prime 2}} D_{U V}^{P T}\left(Q^{\prime 2}\right)
$$

The analytic continuation of $D_{U V}^{P T}\left(Q^{2}\right)$ is now more tricky, since one has to treat differently the two terms on the right hand side of eq.(2.7), namely (since $\Phi_{D}$ is discontinuous) continue $D_{P T}\left(Q^{2}\right)$ with respect to $\Lambda^{2}$ (see previous footnote), and $D_{I R}^{P T}\left(Q^{2}\right)$ with respect to $Q^{2}$ (this is only heuristic, since both terms are separately not well defined). Indeed, the operator $\frac{1}{2 \pi i} \oint_{\left|Q^{\prime 2}\right|=Q^{2}} \frac{d Q^{\prime 2}}{Q^{\prime 2}}$ applied to $D_{I R}^{P T}\left(Q^{2}\right)$ formally reproduces $R_{I R}^{P T}\left(Q^{2}\right)$, as suggested by the previous result. On the other hand, applying this operator to the formal (RS invariant) Borel representation:

$$
D_{P T}\left(Q^{2}\right)=\int_{0}^{\infty} d z \exp \left(-z \beta_{0} \ln \frac{Q^{2}}{\Lambda^{2}}\right) \tilde{D}(z)
$$


yields quite generally:

$$
R_{P T}\left(Q^{2}\right)=\int_{0}^{\infty} d z \exp \left(-z \beta_{0} \ln \frac{Q^{2}}{\Lambda^{2}}\right) \tilde{R}(z)
$$

with:

$$
\tilde{R}(z)=\frac{\sin \left(\pi \beta_{0} z\right)}{\pi \beta_{0} z} \tilde{D}(z)
$$

which is the correct expected relation [23].

A clearer derivation is afforded by using eq.(3.15) and observing that $\dot{\mathcal{F}}_{D, U V}\left(\mu^{2}, Q^{2}\right)$, analytically continued to complex $Q^{2}$, satisfies the relation:

$$
\frac{1}{2 \pi i} \oint_{\left|Q^{\prime 2}\right|=Q^{2}} \frac{d Q^{\prime 2}}{Q^{\prime 2}} \dot{\mathcal{F}}_{D, U V}\left(\mu^{2}, Q^{\prime 2}\right)=\dot{\mathcal{F}}_{R, U V}\left(\mu^{2}, Q^{2}\right)
$$

Eq.(4.14) then follows by applying the operator $\frac{1}{2 \pi i} \oint_{\left|Q^{\prime 2}\right|=Q^{2}} \frac{d Q^{\prime 2}}{Q^{\prime 2}}$ to eq. (3.15) and comparing with eq.(3.9). To check eq.(4.18), I note that:

$$
\dot{\mathcal{F}}_{D, U V}\left(\mu^{2}, Q^{2}\right)=\dot{\mathcal{F}}_{D}\left(\mu^{2} / Q^{2}\right)-\dot{\mathcal{F}}_{D, I R}\left(\mu^{2}, Q^{2}\right)
$$

where:

$$
\dot{\mathcal{F}}_{D, I R}\left(\mu^{2}, Q^{2}\right) \equiv \mu^{2} \int_{0}^{\mu_{I}^{2}} \frac{d k^{2}}{\left(k^{2}+\mu^{2}\right)^{2}} \Phi_{D}\left(k^{2} / Q^{2}\right)
$$

Furthermore $\Phi_{D}$, hence also $\dot{\mathcal{F}}_{D, I R}$, can be analytically continued to complex $Q^{2}$ for large enough $Q^{2} / \mu_{I}^{2}$ (keeping $k^{2}$ and $\mu^{2}$ real), and we have:

$$
\begin{aligned}
\frac{1}{2 \pi i} \oint_{\left|Q^{\prime 2}\right|=Q^{2}} \frac{d Q^{\prime 2}}{Q^{\prime 2}} \dot{\mathcal{F}}_{D, I R}\left(\mu^{2}, Q^{\prime 2}\right) & =\mu^{2} \int_{0}^{\mu_{I}^{2}} \frac{d k^{2}}{\left(k^{2}+\mu^{2}\right)^{2}} \oint_{\left|Q^{\prime 2}\right|=Q^{2}} \frac{d Q^{\prime 2}}{Q^{\prime 2}} \Phi_{D}\left(k^{2} / Q^{\prime 2}\right) \\
& =\mu^{2} \int_{0}^{\mu_{I}^{2}} \frac{d k^{2}}{\left(k^{2}+\mu^{2}\right)^{2}} \Phi_{R}\left(k^{2} / Q^{2}\right)
\end{aligned}
$$

(where I used eq.(4.12)), i.e.:

$$
\frac{1}{2 \pi i} \oint_{\left|Q^{\prime 2}\right|=Q^{2}} \frac{d Q^{\prime 2}}{Q^{\prime 2}} \dot{\mathcal{F}}_{D, I R}\left(\mu^{2}, Q^{\prime 2}\right)=\dot{\mathcal{F}}_{R, I R}\left(\mu^{2}, Q^{2}\right)
$$

which, together with eq.(4.7) and eq.(4.19), and comparing with eq.(3.10), proves eq.(4.18). Note that eq.(4.21) could have been used to derive eq.(4.13) starting from the "Minkowskian representation":

$$
D_{I R}\left(Q^{2}\right)=\int_{0}^{\infty} \frac{d \mu^{2}}{\mu^{2}} \bar{\alpha}_{e f f}\left(\mu^{2}\right) \dot{\mathcal{F}}_{D, I R}\left(\mu^{2}, Q^{2}\right)
$$

and comparing with eq.(3.6).

Finally, a similar relation holds between $\delta D_{U V}$ in eq.(2.5) and the "UV" piece $\delta R_{U V}$ defined by:

$$
\delta R\left(Q^{2}\right) \equiv \delta R_{I R}\left(Q^{2}\right)+\delta R_{U V}\left(Q^{2}\right)
$$


or, equivalently:

$$
R\left(Q^{2}\right) \equiv R_{I R}\left(Q^{2}\right)+R_{U V}^{P T}\left(Q^{2}\right)+\delta R_{U V}\left(Q^{2}\right)
$$

As a consequence of eq.(4.2), (4.13) and (4.14) one indeed gets:

$$
\delta R_{U V}\left(Q^{2}\right)=\frac{1}{2 \pi i} \oint_{\left|Q^{\prime 2}\right|=Q^{2}} \frac{d Q^{\prime 2}}{Q^{\prime 2}} \delta D_{U V}\left(Q^{\prime 2}\right)
$$

However, at the difference of $\delta R_{I R}$ (eq.(2.18)), $\delta R_{U V}$ (and $\delta R$ ) cannot in general be expressed as integrals over $\delta \bar{\alpha}_{s}$ (an exception is $\delta R_{A P T}\left(Q^{2}\right)$, see Appendix $\mathrm{C}$ ). This relation will be used in the next section.

On a more formal level, I note that the present method may be applied to any Minkowskian quantity $R\left(Q^{2}\right)$ (even not related to the discontinuity of a genuine Euclidean correlation function), since one can always associate to any given $R\left(Q^{2}\right)$ a corresponding "Euclidean" $D\left(Q^{2}\right)$ defined by eq.(4.1) and (4.5). This method also shows that the dispersive approach, applied to Minkowskian quantities, can be viewed as an extension of the quark-hadron duality as implemented through finite energy sum rules.

\section{Ultraviolet power corrections}

I now turn to the third, "ultraviolet" contributions in eq.(2.6) and (4.24). These pieces are usually neglected, on the ground they may yield power contributions unrelated to the OPE condensates, and consequently it is often assumed that $\delta \bar{\alpha}_{s}\left(k^{2}\right)$ is very highly suppressed in the UV region. However, as argued in ref.[3, 28], the opposite assumption violates no known principle, and (moderately suppressed) power corrections to $\bar{\alpha}_{s}\left(k^{2}\right)$ are actually quite naturally expected (see section 6). I will discuss two illustrative cases: i) one where these corrections are of IR origin and of the "standard" $1 / k^{4}$ type, and ii) one where one assumes unconventional (i.e. originating from new physics) $1 / k^{2}$ contributions.

i) Let us first assume for the Euclidean quantity $D\left(Q^{2}\right)$ :

$$
\Phi_{D}\left(k^{2} / Q^{2}\right) \simeq A \frac{k^{4}}{Q^{4}}
$$

at small $k^{2}$, corresponding to the standard dimension 4 "gluon condensate", and put:

$$
\Phi_{D}\left(k^{2} / Q^{2}\right) \equiv A \frac{k^{4}}{Q^{4}}+\Phi_{D}^{(3)}\left(k^{2} / Q^{2}\right)
$$

where

$$
\Phi_{D}^{(3)}\left(k^{2} / Q^{2}\right)=\mathcal{O}\left(k^{6} / Q^{6}\right)
$$

at small $k^{2}$. On the other hand at large $k^{2}$ assume (as suggested by the "standard" model of eq.(6.7) below): 


$$
\delta \bar{\alpha}_{s}\left(k^{2}\right) \simeq \frac{c}{\log ^{2} \frac{k^{2}}{\Lambda^{2}}} \frac{\Lambda^{4}}{k^{4}}
$$

Then we have:

$$
\delta D\left(Q^{2}\right)=\int_{0}^{\infty} \frac{d k^{2}}{k^{2}} \delta \bar{\alpha}_{s}\left(k^{2}\right) A \frac{k^{4}}{Q^{4}}+\int_{0}^{\infty} \frac{d k^{2}}{k^{2}} \delta \bar{\alpha}_{s}\left(k^{2}\right) \Phi_{D}^{(3)}\left(k^{2} / Q^{2}\right)
$$

where both integrals are UV convergent ${ }^{6}$. The first integral gives a "gluon condensate" contribution of essentially IR origin (barring the high energy tail):

$$
\int_{0}^{\infty} \frac{d k^{2}}{k^{2}} \delta \bar{\alpha}_{s}\left(k^{2}\right) A \frac{k^{4}}{Q^{4}}=A K \frac{\Lambda^{4}}{Q^{4}}
$$

where:

$$
K \equiv \int_{0}^{\infty} \frac{d k^{2}}{k^{2}} \delta \bar{\alpha}_{s}\left(k^{2}\right) \frac{k^{4}}{\Lambda^{4}}
$$

On the other hand, the second integral yields, at large $Q^{2}$, using eq.(5.4):

$$
\int_{0}^{\infty} \frac{d k^{2}}{k^{2}} \delta \bar{\alpha}_{s}\left(k^{2}\right) \Phi_{D}^{(3)}\left(k^{2} / Q^{2}\right) \simeq c \frac{\Lambda^{4}}{Q^{4}} \int_{0}^{\infty} \frac{d k^{2}}{k^{2}} \frac{Q^{4}}{k^{4}} \Phi_{D}^{(3)}\left(k^{2} / Q^{2}\right) \frac{1}{\log ^{2} \frac{k^{2}}{\Lambda^{2}}}
$$

Noting that $\Phi_{D}\left(k^{2} / Q^{2}\right)$ must vanish at large $k^{2}$ (to insure UV convergence of the defining integral eq.(2.1)), it follows that $\frac{Q^{4}}{k^{4}} \Phi_{D}^{(3)}\left(k^{2} / Q^{2}\right) \rightarrow-A$ for $k^{2} \rightarrow \infty$, which implies the integral on the right hand side of eq.(5.8) is dominated by the UV region, and has the leading behavior at large $Q^{2}$ :

$$
\int_{0}^{\infty} \frac{d k^{2}}{k^{2}} \frac{Q^{4}}{k^{4}} \Phi_{D}^{(3)}\left(k^{2} / Q^{2}\right) \frac{1}{\log ^{2} \frac{k^{2}}{\Lambda^{2}}} \simeq-\frac{A}{\log \frac{Q^{2}}{\Lambda^{2}}}
$$

Eq.(5.9) can be checked by splitting the integral at $k^{2}=Q^{2}$. Note that the integral is IR convergent due to eq.(5.3), and that one gets an $\mathcal{O}\left(1 / \log Q^{2}\right)$ behavior, rather then an $\mathcal{O}\left(1 / \log ^{2} Q^{2}\right)$ one, reflecting the non-vanishing of $\frac{Q^{4}}{k^{4}} \Phi_{D}^{(3)}\left(k^{2} / Q^{2}\right)$ at large $k^{2}$. Hence:

$$
\int_{0}^{\infty} \frac{d k^{2}}{k^{2}} \delta \bar{\alpha}_{s}\left(k^{2}\right) \Phi_{D}^{(3)}\left(k^{2} / Q^{2}\right) \simeq-A c \frac{\Lambda^{4}}{Q^{4}} \frac{1}{\log \frac{Q^{2}}{\Lambda^{2}}}
$$

I stress that this is an ultraviolet correction, insensitive to any IR cutoff $\mu_{I}^{2}$ one might introduce in the integral in eq.(5.10), since the low energy part of the integral below $\mu_{I}^{2}$ contributes a term much smaller then $1 / Q^{4}$ at large $Q^{2}$. One therefore ends up with:

$$
\delta D\left(Q^{2}\right) \simeq A K \frac{\Lambda^{4}}{Q^{4}}\left(1-\frac{c}{K} \frac{1}{\log \frac{Q^{2}}{\Lambda^{2}}}+\ldots\right)
$$

\footnotetext{
${ }^{6}$ The behavior eq.(5.4) is actually the "hardest" one allowing for an UV finite gluon condensate.
} 
It is natural to interpret the logarithmically suppressed term in eq.(5.11) as a contribution to the gluon condensate coefficient function. One then has to face an apparent paradox, since in QCD such a contribution is usually thought to arise from diagrams with two gluon exchanges, with one soft and one hard gluon line, not considered in the present (dressed) single gluon exchange framework. I conclude that the gluon condensate coefficient function computed in the "non-perturbative vacuum" (where non-perturbative contributions to $\bar{\alpha}_{s}$ are taken into account) must differ from the "naive" one computed with standard methods in the "perturbative vacuum". Alternatively, one may adopt the convention to assign the new contribution to the identity operator coefficient function, where it would appear as a power suppressed correction. Such a reshuffling will preserve the property that at least the power series part of the coefficient functions is correctly given by the standard perturbation theory approach. I stress that this unconventional contribution does not really mean new physics (at the difference of the $1 / Q^{2}$ terms to be discussed below), since it is generated by a power suppressed term in $\bar{\alpha}_{s}$ of standard infrared origin. The situation here looks similar to the one in the two dimensional $\mathcal{O}(N)$ non-linear $\sigma$-model, which has been recently reanalysed in the $1 / N$ expansion in [29]. It is thus possible that also in the latter model coefficients functions computed in the perturbative phase may differ ${ }^{7}$ from those computed in the true vacuum.

Eq.(5.11) poses another interesting theoretical problem: one might expect the coefficient $c / K$ to be unambiguous, and all the ambiguity in $\delta D$ to reside in the overall normalization factor $K$ (eq.(5.7)), the "matrix element". The latter is indeed ambiguous if $\bar{\alpha}_{s}^{P T}$, hence $\delta \bar{\alpha}_{s}$, have a (space-like) Landau singularity. Within the present assumptions where $\bar{\alpha}_{s}^{P T}$ has no renormalons ${ }^{8}$ and $c$ is consequently unambiguous, $c / K$ appears however to be ambiguous. One may think of several ways out of this possible inconsistency:

a) One can introduce an IR cut-off $\mu_{I}$ in eq.(5.5) and write eq.(5.11) as:

$$
\delta D\left(Q^{2}\right) \simeq A K_{I R}\left(\mu_{I}\right) \frac{\Lambda^{4}}{Q^{4}}+A K_{U V}\left(\mu_{I}\right) \frac{\Lambda^{4}}{Q^{4}}\left(1-\frac{c}{K_{U V}\left(\mu_{I}\right)} \frac{1}{\log \frac{Q^{2}}{\Lambda^{2}}}+\ldots\right)
$$

where:

$$
\begin{aligned}
K_{I R}\left(\mu_{I}\right) & \equiv \int_{0}^{\mu_{I}^{2}} \frac{d k^{2}}{k^{2}} \delta \bar{\alpha}_{s}\left(k^{2}\right) \frac{k^{4}}{\Lambda^{4}} \\
K_{U V}\left(\mu_{I}\right) & \equiv \int_{\mu_{I}^{2}}^{\infty} \frac{d k^{2}}{k^{2}} \delta \bar{\alpha}_{s}\left(k^{2}\right) \frac{k^{4}}{\Lambda^{4}}
\end{aligned}
$$

with $K_{I R}\left(\mu_{I}\right)+K_{U V}\left(\mu_{I}\right)=K$, and only $K_{I R}\left(\mu_{I}\right)$ is ambiguous.

\footnotetext{
${ }^{7}$ This possibility has not been checked in [29].

${ }^{8}$ It is difficult to see how the ambiguities in $K$ and $c$ could possibly cancell if one makes the alternative assumption that $\bar{\alpha}_{s}^{P T}$ has renormalons.
} 
b) Alternatively, if one does not wish to introduce $\mu_{I}$, one can imagine a split:

$$
\delta \bar{\alpha}_{s}\left(k^{2}\right)=\delta \bar{\alpha}_{s}^{I R}\left(k^{2}\right)+\delta \bar{\alpha}_{s}^{U V}\left(k^{2}\right)
$$

where $\delta \bar{\alpha}_{s}^{I R}$ takes care of the Landau singularity and is confined to the IR region, i.e. decreases very fast at large $k^{2}$, whereas $\delta \bar{\alpha}_{s}^{U V}$ contains no Landau singularity and behaves as $\delta \bar{\alpha}_{s}$ (eq.(5.4)): it is indeed possible to cancell the Landau singularity with a $\delta \bar{\alpha}_{s}^{I R}$ contribution which decreases exponentially at large $k^{2}$ (see Appendix B). Then one can write eq.(5.11) as:

$$
\delta D\left(Q^{2}\right) \simeq A K_{I R} \frac{\Lambda^{4}}{Q^{4}}+A K_{U V} \frac{\Lambda^{4}}{Q^{4}}\left(1-\frac{c}{K_{U V}} \frac{1}{\log \frac{Q^{2}}{\Lambda^{2}}}+\ldots\right)
$$

where:

$$
\begin{aligned}
K_{I R} & \equiv \int_{0}^{\infty} \frac{d k^{2}}{k^{2}} \delta \bar{\alpha}_{s}^{I R}\left(k^{2}\right) \frac{k^{4}}{\Lambda^{4}} \\
K_{U V} & \equiv \int_{0}^{\infty} \frac{d k^{2}}{k^{2}} \delta \bar{\alpha}_{s}^{U V}\left(k^{2}\right) \frac{k^{4}}{\Lambda^{4}}
\end{aligned}
$$

with $K_{I R}+K_{U V}=K$, and only $K_{I R}$ is ambiguous.

c) An attractive third alternative assumes that $K$ is actually unambiguous. This is possible ${ }^{9}$ if $\bar{\alpha}_{s}^{P T}$ turns out to be "causal" (section 3), which implies that $\bar{\alpha}_{s}^{P T}$ and $\delta \bar{\alpha}_{s}$ have no Landau singularities. Note however that in this case $\bar{\alpha}_{s}^{P T}=\bar{\alpha}_{s}^{A P T}$, and consequently $\int_{0}^{\infty} \frac{d k^{2}}{k^{2}} \bar{\alpha}_{s}^{P T}\left(k^{2}\right) \Phi_{D}\left(k^{2} / Q^{2}\right)=D_{A P T}\left(Q^{2}\right)$ differs from the corresponding Borel sum $D_{P T}\left(Q^{2}\right)$ by power terms $\delta D_{A P T}\left(Q^{2}\right)$. In leading order we have, since the coupling is causal (see Appendix B):

$$
\delta D_{A P T}\left(Q^{2}\right) \simeq A K_{A P T} \frac{\Lambda^{4}}{Q^{4}}+\ldots
$$

where $K_{A P T}$ is ambiguous (it cancells the renormalon ambiguity still present [25, 26] in $\left.D_{P T}\left(Q^{2}\right)\right)$. Therefore we end up with:

$$
D\left(Q^{2}\right)=D_{P T}\left(Q^{2}\right)+\delta D\left(Q^{2}\right)
$$

where:

$$
\begin{aligned}
\delta D\left(Q^{2}\right) & \equiv \delta D_{A P T}\left(Q^{2}\right)+\delta D_{N P}\left(Q^{2}\right) \\
& \simeq A K_{A P T} \frac{\Lambda^{4}}{Q^{4}}+A K_{N P} \frac{\Lambda^{4}}{Q^{4}}\left(1-\frac{c}{K_{N P}} \frac{1}{\log \frac{Q^{2}}{\Lambda^{2}}}+\ldots\right)
\end{aligned}
$$

where $\delta D$ and $K$ in eq.(5.11) have been renamed $\delta D_{N P}$ and $K_{N P}$. Note that cases a) and b) are just arbitrary definition-dependent rewritings of eq.(5.11), without

\footnotetext{
${ }^{9} \mathrm{~A}$ weaker, but more artificial, condition is to assume that $\bar{\alpha}_{s}^{P T}$ has only complex [9] Landau singularities.
} 
new physical content. However, all three cases suggest the general ansatz for the $\mathcal{O}\left(1 / Q^{4}\right)$ contribution to have the following "two-component" form, once "standard" corrections due to double gluon exchange are taken into account:

$$
\begin{aligned}
\delta D\left(Q^{2}\right) & \simeq A K_{P T} \frac{\Lambda^{4}}{Q^{4}}\left(1+d_{P T} \frac{1}{\log \frac{Q^{2}}{\Lambda^{2}}}+\ldots\right) \\
& +A K_{N P} \frac{\Lambda^{4}}{Q^{4}}\left(1+\left(d_{P T}+d_{N P}\right) \frac{1}{\log \frac{Q^{2}}{\Lambda^{2}}}+\ldots\right)
\end{aligned}
$$

where the first contribution contains the ambiguity through the constant $K_{P T}$ and has the standard perturbative coefficient $d_{P T}$, while the second contribution arises in the non-perturbative vacuum, with $d_{N P}=-c / K_{N P}$. Since $K_{P T}$ cancells the renormalon ambiguity, which depends on information contained in perturbation theory, the first contribution can be thought of being of a "perturbative" nature, at the difference of the second one, which depends on a more genuinely non-perturbative information. The different logarithmic corrections in eq.(5.19) should allow to separate unambiguously the two contributions.

ii) Let us next consider the case where there is a leading $\mathcal{O}\left(1 / Q^{2}\right)$ power correction of UV origin. For the Euclidean quantity $D\left(Q^{2}\right)$, this means [10] that $\left|\Phi_{D}\left(k^{2} / Q^{2}\right)\right| \ll\left|\frac{k^{2}}{Q^{2}}\right|$ at small $k^{2}$, while $\delta \bar{\alpha}_{s}\left(k^{2}\right)$ is $\mathcal{O}\left(1 / k^{2}\right)$ at large $k^{2}$, so that the leading IR power correction is parametrically suppressed compared to the UV one. For instance, if one assumes:

$$
\delta \bar{\alpha}_{s}\left(k^{2}\right) \simeq b_{1} \frac{\Lambda^{2}}{k^{2}}
$$

and substitute into $\delta D_{U V}$ (eq.(2.5)), one gets at large $Q^{2}$ :

$$
\delta D_{U V}\left(Q^{2}\right) \simeq A_{D} b_{1} \frac{\Lambda^{2}}{Q^{2}}
$$

with:

$$
A_{D} \equiv \int_{0}^{\infty} \frac{d k^{2}}{k^{2}} \frac{Q^{2}}{k^{2}} \Phi_{D}\left(k^{2} / Q^{2}\right)
$$

where the integral is IR convergent from the stated assumptions. Note the same assumption on $\Phi_{D}$ implies, expanding at small $\mu^{2}$ under the integral in eq.(3.14):

$$
\dot{\mathcal{F}}_{D}\left(\mu^{2} / Q^{2}\right) \simeq A_{D} \frac{\mu^{2}}{Q^{2}}
$$

i.e. an analytic small $\mu^{2}$ behavior.

The remarks in [10] can be generalized to Minkowskian quantities $R\left(Q^{2}\right)$. Let us similarly assume for small $\mu^{2}$ :

$$
\dot{\mathcal{F}}_{R}\left(\mu^{2} / Q^{2}\right) \simeq A_{R} \frac{\mu^{2}}{Q^{2}}
$$


while, at large $k^{2}$, allowing for a logarithmic correction for the sake of generality:

$$
\delta \bar{\alpha}_{s}\left(k^{2}\right) \simeq\left(b_{1}+\frac{c_{1}}{\log \frac{k^{2}}{\Lambda^{2}}}\right) \frac{\Lambda^{2}}{k^{2}}
$$

where $b_{1}$ and $c_{1}$ are non-perturbative parameters (this is only an illustrative example, since no theory presently exists for these "unorthodox" power corrections).

A derivation of the analogue of eq.(5.21) based on the results of Appendix C (e.g. eq.(2.16), or eq.(C.12)) is possible, but cumbersome, since one has to introduce the split eq.(B.7), and also write down dispersion relations which usually involve subtraction constants, whereas only the assumption eq.(5.24) is really needed. In particular, the constant $A_{R}$ is equal to the subtraction constant $a_{0}$, and is independent of $\Phi_{R}$, if eq.(C.6) is assumed; then the leading UV power correction is given by the first, "subtraction" term on the right hand side of eq.(C.12), which yields a result similar to eq.(5.21). Alternatively, one could use [3] the expression for power corrections in term of the Minkowkian coupling $\bar{\alpha}_{e f f}$ (see Appendix B), but this approach is unconvenient too, since it again relies on the split eq.(B.7).

All these problems are however circumvented if one deals first with the associated Euclidean quantity $D\left(Q^{2}\right)$, and deduce the corresponding power corrections for $R\left(Q^{2}\right)$ using analyticity (eq.(4.25)). Indeed eq.(4.5) and eq.(5.24) imply the analytic small $k^{2}$ behavior of the Euclidean kernel $\Phi_{D}$ :

$$
\Phi_{D}\left(k^{2} / Q^{2}\right) \simeq A_{R} \frac{k^{2}}{Q^{2}}
$$

(the corresponding $\dot{\mathcal{F}}_{D}$ behaves as $A_{R} \frac{\mu^{2}}{Q^{2}} \log \frac{Q^{2}}{\mu^{2}}$ at small $\mu^{2}$, and signals the contribution of a $\mathrm{d}=2$ operator in the OPE of the considered Euclidean correlation function: this is why eq.(5.24) does not hold for e.g. $R_{e^{+} e^{-}}$). One can then show (see Appendix A), at large $Q^{2}$ :

$$
\delta D_{U V}\left(Q^{2}\right) \simeq \frac{\Lambda^{2}}{Q^{2}}\left[A_{R}\left(b_{1} \log \frac{Q^{2}}{\Lambda^{2}}+c_{1} \log \log \frac{Q^{2}}{\Lambda^{2}}\right)+\text { const }+\mathcal{O}\left(\frac{1}{\log \frac{Q^{2}}{\Lambda^{2}}}\right)\right]
$$

where the constant and $\mathcal{O}\left(1 / \log Q^{2}\right)$ terms cannot be expressed only in term of the small $k^{2}$ behavior of $\Phi_{D}$, and the first two terms on the right-hand side represent also the leading contributions to $\delta D$, and are of ultraviolet origin $(\delta D$ contains in addition an $\mathcal{O}\left(1 / Q^{2}\right)$ piece of IR origin, coming from the first term in eq.(2.8)) . Applying the operator $\frac{1}{2 \pi i} \oint_{\left|Q^{\prime 2}\right|=Q^{2}} \frac{d Q^{\prime 2}}{Q^{\prime 2}}$ to the right-hand side of eq.(5.27) then yields, as a consequence of eq.(4.25), the leading contribution to $\delta R_{U V}\left(\right.$ and $\delta R$ ) at large $Q^{2}$ :

$$
\delta R_{U V}\left(Q^{2}\right) \simeq \frac{\Lambda^{2}}{Q^{2}}\left[A_{R}\left(b_{1}+\frac{c_{1}}{\log \frac{Q^{2}}{\Lambda^{2}}}\right)+\mathcal{O}\left(\frac{1}{\log ^{2} \frac{Q^{2}}{\Lambda^{2}}}\right)\right]
$$


where the $\mathcal{O}\left(1 / \log ^{2} Q^{2}\right)$ term cannot be expressed only in term of the small $\mu^{2}$ behavior of $\dot{\mathcal{F}}_{R}$. As a check, eq.(5.27) can also be obtained directly from eq.(5.28) and the dispersion relation eq.(4.1) using similar methods as in Appendix A. Eq.(5.28), which is the analogue of eq.(5.21), is naturally identified as an ultraviolet contribution; note in this respect that the leading IR power contribution originating from the term $D_{I R}\left(Q^{2}\right)$ in eq.(2.6) is a pure power, if $\Phi_{D}$ is analytic at small $k^{2}$, hence gives no contribution to $R$ after applying the operator $\frac{1}{2 \pi i} \oint_{\left|Q^{\prime 2}\right|=Q^{2}} \frac{d Q^{\prime 2}}{Q^{\prime 2}}$.

One can then relate simply the UV power corrections in different channels, if the non-perturbative parameters $b_{1}$ and $c_{1}$, which have to be fitted, are assumed to be universal (like the "physical" coupling $\bar{\alpha}_{s}$ itself), since the channel-dependant parameter $A_{R}$ is calculable from Feynman diagrams. These remarks lead to a simple phenomenology [10] of $1 / Q^{2}$ terms (if they turn out large enough to be detected). The channel dependence may be sizable. For instance, writing a generic Euclidean correlation function computed at one loop with a gluon of mass $\mu^{2} \operatorname{as}^{10}$ :

$$
D\left(Q^{2}\right)=1+\frac{\alpha_{s}}{\pi}\left[\mathcal{F}_{D}(0)-A_{D} \frac{\mu^{2}}{Q^{2}}+\ldots\right]
$$

(where the parton model contribution is normalized to unity), one has [15]:

$$
A_{\text {vector }}=\frac{8}{3}(4-3 \zeta(3)) \simeq 1.07
$$

for the vector correlation function, whereas [30]:

$$
A_{\text {pseudoscalar }}=4
$$

for the pseudoscalar correlation function ${ }^{11}$ (after taking two derivatives [31] to get rid of an overall UV divergence and extracting two powers of quark masses). It is thus possible that eventual $1 / Q^{2}$ terms (whatever their physical origin) may have more important effects in the pseudoscalar channel [32] then in the vector one (where they seem to be negligible [33]). Unfortunately, a phenomenological determination of such terms may be difficult given the large perturbative corrections found in the pseudoscalar channel.

Currents with anomalous dimensions: in the pseudoscalar case (and in many other), the corresponding current has anomalous dimension. Here I suggest an ansatz to deal with the latter. It is useful to factor out explicitly the anomalous dimension dependence using the renormalization group, and write the correlation function as:

$$
D\left(Q^{2}\right)=\frac{D_{i n v}\left(Q^{2}\right)}{Z\left(\mu^{2}\right)}
$$

\footnotetext{
${ }^{10}$ The normalization of $\mathcal{F}_{D}$ here is different from the one in eq.(3.13).

${ }^{11} \mathrm{~A}$ closely related result is quoted in $[1,32]$.
} 
where $D_{i n v}\left(Q^{2}\right)$ is renormalization group invariant, and $Z\left(\mu^{2}\right)$ is the integrated anomalous dimension factor, related to the anomalous dimension function $\gamma\left(\alpha_{s}\right)$ by:

$$
\frac{d \log Z}{d \log \mu^{2}}=\gamma\left(\alpha_{s}\left(\mu^{2}\right)\right)
$$

In perturbation theory, we have:

$$
D_{i n v}^{P T}\left(Q^{2}\right)=\left(\alpha_{s}\right)^{\gamma_{0} / \beta_{0}}\left(1+\mathcal{O}\left(\alpha_{s}\right)\right)
$$

where $\gamma_{0}$ is the one loop anomalous dimension, and similarly for $Z\left(\mu^{2}\right)$. It is convenient to introduce the "effective charges" [34]:

$$
D_{i n v}\left(Q^{2}\right) \equiv\left[A\left(Q^{2}\right)\right]^{\gamma_{0} / \beta_{0}}
$$

and similarly

$$
Z\left(\mu^{2}\right) \equiv\left[a\left(\mu^{2}\right)\right]^{\gamma_{0} / \beta_{0}}
$$

where both $A\left(Q^{2}\right)$ and $a\left(\mu^{2}\right)$ are $\mathcal{O}\left(\alpha_{s}\right)$ quantities. Then:

$$
D\left(Q^{2}\right)=\left[\frac{A\left(Q^{2}\right)}{a\left(\mu^{2}\right)}\right]^{\gamma_{0} / \beta_{0}}
$$

Let us now assume that an $\mathcal{O}\left(1 / Q^{2}\right)$ correction appears in the "non-perturbative" $\mathrm{RG}$ invariant correlation function in the form:

$$
D_{i n v}\left(Q^{2}\right)=D_{i n v}^{P T}\left(Q^{2}\right)\left(1+\frac{\gamma_{0}}{\beta_{0}} \frac{C}{Q^{2}}+\ldots\right)
$$

which implies:

$$
A\left(Q^{2}\right)=A_{P T}\left(Q^{2}\right)\left(1+\frac{C}{Q^{2}}+\ldots\right)
$$

whereas no such corrections are expected in $Z\left(\mu^{2}\right)$ and $a\left(\mu^{2}\right)$, which are entirely perturbative quantities. Then we have at large $Q^{2}$ :

$$
\log \left[\frac{A\left(Q^{2}\right)}{a\left(\mu^{2}\right)}\right]=\log \left[\frac{A_{P T}\left(Q^{2}\right)}{a\left(\mu^{2}\right)}\right]+\frac{C}{Q^{2}}+\ldots
$$

Note that $\log \left[\frac{A_{P T}\left(Q^{2}\right)}{a\left(\mu^{2}\right)}\right]$ is an $\mathcal{O}\left(\alpha_{s}\right)$ quantity ${ }^{12}$. In order to make contact between this quantity and the single gluon exchange integral, I now appeal to the large $N_{f}$

\footnotetext{
${ }^{12}$ It is interesting to note that $\log \left[A_{P T}\left(Q^{2}\right)\right]$ corresponds formally to the image in coupling constant space of the "bare Borel transform" of [15]: the logarithmic dependence on the coupling simply reflects the singular behavior of the "bare Borel transform" at the origin. Similarly, $\log \left[\frac{A_{P T}\left(Q^{2}\right)}{a\left(\mu^{2}\right)}\right]$ is essentially the image of the corresponding renormalized Borel transform (see Appendix A in [15]).
} 
limit, which displays the corresponding single renormalon chain type of diagrams. In this limit eq.(5.37) yields, since $\gamma_{0} / \beta_{0}$ is $\mathcal{O}\left(1 / N_{f}\right)$ :

$$
D\left(Q^{2}\right)=1+\frac{\gamma_{0}}{\beta_{0}} \log \left[\frac{A\left(Q^{2}\right)}{a\left(\mu^{2}\right)}\right]_{\mid N_{f}=\infty}+\mathcal{O}\left(1 / N_{f}^{2}\right)
$$

which shows that $\frac{\gamma_{0}}{\beta_{0}} \log \left[\frac{A_{P T}\left(Q^{2}\right)}{a\left(\mu^{2}\right)}\right]_{\mid N_{f}=\infty}$ should be identified to the sum of the single renormalon chain diagrams. Consequently, the power correction eq.(5.21) obtained from these diagrams should be identified to $\frac{\gamma_{0}}{\beta_{0}} \frac{C}{Q^{2}}$, i.e. we have:

$$
\frac{\gamma_{0}}{\beta_{0}} C=A_{D} b_{1}
$$

\section{Ansätze for the non-perturbative contributions to the uni- versal QCD running coupling}

\subsection{QED inspired models}

Although they are usually completely neglected, power suppressed corrections to a "physical" coupling such as $\bar{\alpha}_{s}$, which is supposed to be defined also at the nonperturbative level (and is assumed in [4] to be the universal coupling of QCD) are a natural expectation, and could eventually be derived from the OPE itself as the following QED analogy shows. In QED, the coupling $\bar{\alpha}_{s}\left(k^{2}\right)$ should be identified, in the present dressed single gluon exchange context, to the Gell-Mann-Low effective charge $\bar{\alpha}$, related to the photon vacuum polarisation $\Pi\left(k^{2}\right)$ by:

$$
\bar{\alpha}\left(k^{2}\right)=\frac{\alpha}{1+\alpha \Pi\left(k^{2} / \mu^{2}, \alpha\right)}
$$

One expects $\Pi\left(k^{2}\right)$, hence $\bar{\alpha}\left(k^{2}\right)$, to receive power contributions from the OPE. Of course, this cannot happen in QED itself, which is an IR trivial theory, but might occur in the "large $\beta_{0}$ ", $N_{f}=-\infty$ limit of QCD . Instead of $\Pi\left(k^{2}\right)$, it is convenient to introduce the related (properly normalized) renormalization group invariant "Adler function" (with the Born term removed):

$$
\mathcal{A}\left(k^{2}\right)=\frac{1}{\beta_{0}}\left(\frac{d \Pi}{d \log k^{2}}-\frac{d \Pi}{d \log k^{2}} \mid \alpha=0\right)
$$

$\mathcal{A}_{s}$, its assumed analogue in QCD, contributes the higher order terms in the renormalization group equation:

$$
\frac{d \bar{\alpha}_{s}}{d \ln k^{2}}=-\beta_{0}\left(\bar{\alpha}_{s}\right)^{2}\left(1+\mathcal{A}_{s}\right)
$$


Consider now the $N_{f}=-\infty$ limit in QCD. Then $\mathcal{A}_{s}\left(k^{2}\right)$ is expected to be purely non-perturbative, since in this limit the perturbative part of $\bar{\alpha}_{s}$ is just the oneloop coupling $\bar{\alpha}_{s}^{P T}\left(k^{2}\right)=1 / \beta_{0} \ln \left(k^{2} / \Lambda^{2}\right)$. Indeed, OPE-renormalons type arguments suggest the general structure [35] at large $k^{2}$ :

$$
\mathcal{A}_{s}\left(k^{2}\right)=\sum_{p=1}^{\infty}\left(a_{p} \log \frac{k^{2}}{\Lambda^{2}}+b_{p}\right)\left(\frac{\Lambda^{2}}{k^{2}}\right)^{p}
$$

where the log enhanced power corrections reflect the presence of double IR renormalons poles [36]. Eq.(6.3) with $\mathcal{A}_{s}$ as in eq.(6.4) can be easily integrated to give:

$$
\frac{1}{\beta_{0} \bar{\alpha}_{s}}=\log \frac{k^{2}}{\Lambda^{2}}-\sum_{p=1}^{\infty} \frac{1}{p}\left(\frac{\Lambda^{2}}{k^{2}}\right)^{p}\left(a_{p} \log \frac{k^{2}}{\Lambda^{2}}+b_{p}+\frac{a_{p}}{p}\right)
$$

which yields the expansion:

$$
\bar{\alpha}_{s}\left(k^{2}\right)=\bar{\alpha}_{s}^{P T}\left(k^{2}\right)+\sum_{p=1}^{\infty}\left[c_{p, 0} \bar{\alpha}_{s}^{P T}\left(k^{2}\right)+\ldots .+c_{p, p}\left(\bar{\alpha}_{s}^{P T}\left(k^{2}\right)\right)^{p+1}\right]\left(\frac{\Lambda^{2}}{k^{2}}\right)^{p}
$$

where the first terms are given by:

$$
\left\{\begin{array}{l}
c_{1,0}=a_{1} \\
c_{1,1}=\beta_{0}\left(a_{1}+b_{1}\right)
\end{array}\right.
$$

and:

$$
\left\{\begin{array}{l}
c_{2,0}=\frac{1}{2} a_{2}+a_{1}^{2} \\
c_{2,1}=\beta_{0}\left(\frac{1}{4} a_{2}+\frac{1}{2} b_{2}+2 a_{1} b_{1}+2 a_{1}^{2}\right) \\
c_{2,2}=\left(\beta_{0}\right)^{2}\left(a_{1}+b_{1}\right)^{2}
\end{array}\right.
$$

In QCD, one actually expects : $a_{1}=b_{1}=0$ (reflecting the absence of $d=2$ gauge invariant operator), and $a_{2}=0$ (reflecting the absence of anomalous dimension in the gluon condensate), which gives:

$$
\bar{\alpha}_{s}\left(k^{2}\right)=\bar{\alpha}_{s}^{P T}\left(k^{2}\right)+\frac{1}{2} \beta_{0} b_{2}\left(\bar{\alpha}_{s}^{P T}\left(k^{2}\right)\right)^{2} \frac{\Lambda^{4}}{k^{4}}+\ldots
$$

i.e. in this semi-standard framework there is no (as expected) $1 / k^{2}$ correction. It is interesting to note that keeping only the $p=2$ (gluon condensate) contribution in eq.(6.4) with $a_{2}=0$ yields:

$$
\bar{\alpha}_{s}\left(k^{2}\right)=\frac{1}{\beta_{0}\left(\ln \frac{k^{2}}{\Lambda^{2}}-\frac{b_{2}}{2} \frac{\Lambda^{4}}{k^{4}}\right)}
$$

This model ${ }^{13}$ coincides with a previously suggested ansatz [37] based on different arguments, which lead to the suggestion that the running coupling should satisfy the

\footnotetext{
${ }^{13} \mathrm{It}$ is amusing to note that the popular Richardson coupling: $\bar{\alpha}_{s}\left(k^{2}\right)=1 / \beta_{0} \log \left(c^{2}+k^{2} / \Lambda^{2}\right)$ is also very simply described by equation (6.3). It corresponds to: $\mathcal{A}_{s}\left(k^{2}\right)=-1 /\left(1+k^{2} / c^{2} \Lambda^{2}\right)$ i.e. to $\mathcal{A}_{s}$ given by a simple pole in the time-like region, with prescribed residue.
} 
second order differential equation:

$$
\frac{1}{2}\left(\frac{1}{\bar{\alpha}_{s}}\right)^{\prime \prime}+\left(\frac{1}{\bar{\alpha}_{s}}\right)^{\prime}=\beta_{0}
$$

(where ${ }^{\prime} \equiv d / d \log k^{2}$ ) whose solution turns out to be given by eq.(6.8). A possibly welcome feature [38] of the coupling eq.(6.8) is that it vanishes at $k^{2}=0$. However, it probably also has Landau singularities on the first sheet of the complex $k^{2}$ plane, and does not satisfy the dispersion relation eq.(2.13).

It is tempting to speculate that the series in eq.(6.4) has a finite convergence radius. A general ansatz for $\bar{\alpha}_{s}$ would then be given by the solution $\bar{\alpha}_{s}^{U V}$ of eq.(6.3) (i.e. the coupling as reconstructed from its short distance expansion), augmented by a term $\delta \bar{\alpha}_{s}^{I R}$ whose support is entirely in the infrared region (i.e. has an exponentially supressed UV tail), both pieces being assumed to satisfy the dispersion relation eq.(2.13):

$$
\bar{\alpha}_{s}\left(k^{2}\right)=\bar{\alpha}_{s}^{U V}\left(k^{2}\right)+\delta \bar{\alpha}_{s}^{I R}\left(k^{2}\right)
$$

If it were possible to know $\bar{\alpha}_{s}^{U V}$ analytically, then the introduction of an IR cut-off $\mu_{I}$ in eq.(2.4) would not be anymore necessary, ${ }^{14}$ and one could parametrize the remaining IR contributions with (UV convergent) moments of the $\delta \bar{\alpha}_{s}^{I R}$ piece, along the lines of [4].

\subsection{Models based on the "analytic perturbation theory" coupling}

The "analytic perturbation theory" (APT) coupling [24, 3, 9, 15]:

$$
\bar{\alpha}_{s}^{A P T}\left(k^{2}\right) \equiv k^{2} \int_{0}^{\infty} \frac{d \mu^{2}}{\left(\mu^{2}+k^{2}\right)^{2}} \bar{\alpha}_{e f f}^{P T}\left(\mu^{2}\right)
$$

is used in Appendices B and $\mathrm{C}$ for entirely technical reasons, to deal with power corrections in the "Minkowskian representation". It is nevertheless tempting to speculate about its eventual physical relevance (some remarkable infrared properties have been pointed out in $[24,9]$ ). Since it is always possible to perform the split eq.(B.7):

$$
\bar{\alpha}_{s}\left(k^{2}\right)=\bar{\alpha}_{s}^{A P T}\left(k^{2}\right)+\delta \bar{\alpha}_{s}^{A N P}\left(k^{2}\right)
$$

which is just a definition, physical content arises only if additional assumptions are made concerning $\delta \bar{\alpha}_{s}^{A N P}$. Two simple possibilities ${ }^{15}$ come to mind:

\footnotetext{
${ }^{14}$ If it further happens that $\bar{\alpha}_{s}^{U V}$ has its support in the ultraviolet region and is exponentially suppressed in the infrared, for instance $\bar{\alpha}_{s}^{U V}\left(k^{2}\right)=\exp \left[-\left(\Lambda^{2} / k^{2}\right)^{N}\right] 1 / \beta_{0} \log \left(c^{2}+k^{2} / \Lambda^{2}\right)$ (this example satisfies eq.(6.3) and (6.4)), then the ansatz eq.(6.10) would be a natural and unique smooth-out substitute of the introduction of a sharp IR cut-off (eq.(2.4)), which amounts to write: $\bar{\alpha}_{s}=\bar{\alpha}_{s} \theta\left(k^{2}-\mu_{I}^{2}\right)+\bar{\alpha}_{s} \theta\left(\mu_{I}^{2}-k^{2}\right)$ where the first and second term play the role respectively of $\bar{\alpha}_{s}^{U V}$ and $\delta \bar{\alpha}_{s}^{I R}$. Taking e.g. $\delta \bar{\alpha}_{s}^{I R}\left(k^{2}\right)=C \exp \left(-k^{2} / \Lambda^{2}\right)$ (this choice is not quite satisfactory, since it violates asymptotic freedom for $\left.\operatorname{Re}\left(k^{2}\right)<0\right)$ ), eq.(6.10) reproduces (for $C=-1$ and $N=1$ ) an example given in [31] (with a different motivation).

${ }^{15}$ For another suggestion see [39].
} 
i) One can assume [3] that $\delta \bar{\alpha}_{s}^{A N P}\left(k^{2}\right) \equiv \delta \bar{\alpha}_{s}^{I R}\left(k^{2}\right)$ is an essentially infrared contribution, highly suppressed in the ultraviolet region: $\bar{\alpha}_{s}^{A P T}$ thus plays the role ${ }^{16}$ of $\bar{\alpha}_{s}^{U V}$ in eq.(6.10). This assumption leads, unless $\bar{\alpha}_{s}^{P T}$ is causal, to the prediction of unconventional, OPE unrelated $1 / Q^{2}$ power terms, which (when calculated with a one-loop model) seem to predict [3] a too large value of $\alpha_{s}$ in $\tau$-decay. One also obtains the wrong sign [28] for the detected [40] $1 / Q^{2}$ term to the lattice gluon condensate. These problems are avoided, as mentionned above, if one assumes that $\bar{\alpha}_{s}^{P T}$ is causal, i.e. that $\bar{\alpha}_{s}^{P T}=\bar{\alpha}_{s}^{A P T}$, which is a natural possibility in the framework of [4] (see also the discussion in section 5).

ii) Alternatively, one can make the opposite assumption that $\delta \bar{\alpha}_{s}^{A N P}\left(k^{2}\right) \equiv$ $\delta \bar{\alpha}_{s}^{U V}\left(k^{2}\right)$ is an essentially ultraviolet contribution, highly suppressed in the infrared region (e.g. $\left.\delta \bar{\alpha}_{s}^{U V}\left(k^{2}\right)=C \exp \left[-\left(\Lambda^{2} / k^{2}\right)^{N}\right] \frac{\Lambda^{2}}{k^{2}}\right)$. This assumption implies that the infrared part of the total $\bar{\alpha}_{s}$ coupling is essentially given by that of $\bar{\alpha}_{s}^{A P T}$, while making no commitment upon the magnitude and sign of eventual $1 / Q^{2}$ terms. Furthermore, it turns out that the low energy behavior of the APT coupling is rather insensitive $[24,9]$ to higher loops in the perturbative beta function, and close to the one-loop APT model (eq.(B.5)), for a large class of renormalization schemes (the important exception [9] to the previous statement is again the case where $\bar{\alpha}_{s}^{P T}$ is causal). Thus, unless $\bar{\alpha}_{s}^{P T}$ is causal, $R_{I R}\left(Q^{2}\right)$ (eq.(2.19)) will be approximated by:

$$
R_{I R}\left(Q^{2}\right) \simeq \int_{0}^{\mu_{I}^{2}} \frac{d k^{2}}{k^{2}} \bar{\alpha}_{s}^{A P T}\left(k^{2}\right) \Phi_{R}\left(k^{2} / Q^{2}\right) \simeq \int_{0}^{\mu_{I}^{2}} \frac{d k^{2}}{k^{2}} \bar{\alpha}_{s}^{A P T}\left(k^{2}\right)_{\mid \text {one-loop }} \Phi_{R}\left(k^{2} / Q^{2}\right)
$$

i.e. an essentially parameter free (apart from $\mu_{I}$ ) prediction! There may be some preliminary phenomenological evidence [24] in favor of such an assumption (see however [41] for a possible theoretical inconsistency).

\section{Scheme dependence issues}

In standard applications, one neglects UV power corrections, and uses eq.(2.21):

$$
R\left(Q^{2}\right) \simeq R_{P T}\left(Q^{2}\right)+\int_{0}^{\mu_{I}^{2}} \frac{d k^{2}}{k^{2}} \bar{\alpha}_{s}\left(k^{2}\right) \Phi_{R}\left(k^{2} / Q^{2}\right)-\int_{0}^{\mu_{I}^{2}} \frac{d k^{2}}{k^{2}} \bar{\alpha}_{s}^{P T}\left(k^{2}\right) \Phi_{R}\left(k^{2} / Q^{2}\right)
$$

where $R_{P T}\left(Q^{2}\right)$ is now meant to be the full available (usually next to leading order) perturbative QCD expression for $R$, and is not restricted to be given by the single gluon exchange expression eq.(3.4) (contrary to the two power corrections integrals in eq.(7.1)). This procedure however suffers from the usual scheme dependence ambiguity concerning the choice of renormalization scheme (RS) and scale parameter in the trunkated expression for $R_{P T}\left(Q^{2}\right)$. This problem becomes severe if next to leading order corrections are large with the usual choice of RS (this happens in particular in

\footnotetext{
${ }^{16}$ However the corresponding $\mathcal{A}_{s}\left(k^{2}\right)$ does not have the structure of eq.(6.4).
} 
the case of thrust [5], where these corrections in the $\overline{M S}$ scheme with the standard choice of scale are close to $40 \%$ at the $\mathrm{Z}$ mass). A resummation of such large corrections appears necessary. One reasonable procedure for doing so, in absence of any other information, is the "effective charge" scheme [34]. It has recently been applied [42] to the case of thrust, where it yields results similar to those obtained with a "low $\mu$ " choice [43] of renormalization point, close to the one $(\mu=0.08 Q)$ which sets to zero the next to leading order corrections. The effect of these alternative procedures is to drastically reduce the size of the $1 / Q$ power term needed to fit the data. It is clearly an important issue to determine the correct $\mathrm{RS}$.

However, one should remark that if one sticks to the single gluon exchange picture even for $R_{P T}\left(Q^{2}\right)$, there is no real scheme dependence issue anymore, since the physical universal running coupling $\bar{\alpha}_{s}^{P T}\left(k^{2}\right)$ which dresses the gluon propagator used under the single gluon exchange integral has already been uniquely identified (up to next to leading order in the $\overline{M S}$ scheme). This is clear ${ }^{17}$ for an Euclidean quantity, where one can write (eq.(2.6)), neglecting UV power corrections:

$$
D\left(Q^{2}\right) \simeq \int_{0}^{\mu_{I}^{2}} \frac{d k^{2}}{k^{2}} \bar{\alpha}_{s}\left(k^{2}\right) \Phi_{D}\left(k^{2} / Q^{2}\right)+\int_{\mu_{I}^{2}}^{\infty} \frac{d k^{2}}{k^{2}} \bar{\alpha}_{s}^{P T}\left(k^{2}\right) \Phi_{D}\left(k^{2} / Q^{2}\right)
$$

and $\bar{\alpha}_{s}^{P T}\left(k^{2}\right)$ can be unambiguously determined in an RS invariant manner (in term of e.g. $\left.\Lambda_{\overline{M S}}\right)$ for all scales above the IR cut-off $\mu_{I}$ by integrating its own renormalization group equation (known presently only up to the first two (universal) loops). Note that the IR cut-off $\mu_{I}$ alleviates the problem of integrating over the Landau singularity in the second integral (the "regularized perturbation theory" piece).

For Minkowskian quantities, where an integral representation in term of $\bar{\alpha}_{s}\left(k^{2}\right)$ is not available, one has to use the (RS invariant) Borel transform formalism of section 3 , i.e. write (eq.(2.21)):

$$
\begin{aligned}
R\left(Q^{2}\right) & \simeq \int_{0}^{\mu_{I}^{2}} \frac{d k^{2}}{k^{2}} \bar{\alpha}_{s}\left(k^{2}\right) \Phi_{R}\left(k^{2} / Q^{2}\right) \\
& +\int_{0}^{\infty} d z \tilde{\alpha}_{e f f}(z)\left[\int_{0}^{\infty} \frac{d \mu^{2}}{\mu^{2}} \dot{\mathcal{F}}_{R, U V}\left(\mu^{2}, Q^{2}\right) \exp \left(-z \beta_{0} \ln \frac{\mu^{2}}{\Lambda^{2}}\right)\right]
\end{aligned}
$$

where the second integral is the perturbative part of the Beneke-Braun like "gluon mass" integral eq.(3.12) (but with the "IR regularized" characteristic function replacing the full characteristic function). It would be interesting to establish the effect [44] of using eq.(7.3) on the size of the $1 / Q$ term in the thrust case. The crucial question underlying the reliability of this method is whether the alleged QCD "dressed skeleton expansion", trunkated at its first term (the single gluon exchange level) is a good approximation.

\footnotetext{
${ }^{17}$ An analoguous observation has been made before [12] as the essential RS invariance of the QED "dressed skeleton expansion"; it is also closely related to the intuition behind the BLM scheme [11].
} 


\section{Summary and Conclusions}

Power corrections to generic QCD Minkowskian observables have been discussed in the "dressed single gluon exchange approximation", assuming the existence of a (universal) QCD coupling $\bar{\alpha}_{s}$ defined at the non-perturbative level, and regular in the infrared region. Following [5], one introduces an IR cut-off $\mu_{I}$, and distinguish between IR and UV power contributions. This procedure appears hardly avoidable in practice: the alternative approach based on the split eq.(6.10) $\bar{\alpha}_{s}=\bar{\alpha}_{s}^{U V}+\delta \bar{\alpha}_{s}^{I R}$ (where $\delta \bar{\alpha}_{s}^{I R}$ is restricted to the infrared region) requires a reconstruction of the "correct" unique short distance coupling $\bar{\alpha}_{s}^{U V}$ from the short distance expansion of $\bar{\alpha}_{s}$, which is very hard to achieve (assuming it can be done at all), since one may have to sum an infinite set of power suppressed corrections: $\bar{\alpha}_{s}^{U V}$ cannot be given only by the perturbative part $\bar{\alpha}_{s}^{P T}$ which has a Landau singularity, unless $\bar{\alpha}_{s}^{P T}$ is causal. The adopted procedure allows a parametrization of IR power corrections in terms of low energy moments of the full coupling $\bar{\alpha}_{s}$, which form one set of (universal) non-perturbative parameters. On the other hand, UV power corrections rely on the split of eq.(2.2) between perturbative and non-perturbative contributions to $\bar{\alpha}_{s}$ : the other set of (universal) non-perturbative parameters are those which occur (assuming a simple form) in the high energy expansion of the non-perturbative part $\delta \bar{\alpha}_{s}$, i.e. power corrections to the coupling itself. I argued that the latter are a natural expectation for a coupling such as $\bar{\alpha}_{s}$ assumed to be defined at the non-perturbative level, and discussed some simple models for them which do not necessarily imply new physics. However, I argued that in general "physical" models for the universal coupling are not expected to have too highly suppressed corrections to asymptotic freedom. Consequently, the coefficients functions of higher dimensional operators computed in the non-perturbative vacuum may differ from the standard ones computed in the perturbative vacuum, even in the standard OPE framework where all power corrections are ultimately of IR origin.

The basic diagrammatic quantity which, together with the previous parameters, determine the power corrections to a given process is the "gluon-mass" dependent characteristic function. Its discontinuity controls the IR power corrections, which are therefore related to non-analytic terms in the small gluon mass expansion, whereas both the analytic and the non-analytic terms in this expansion control the UV power corrections. Furthermore, I have checked that IR renormalons cancell once the IR part of the perturbative calculation is properly removed; the relevant diagrammatic object is the "IR cut-off" characteristic function. To establish these properties for Minkowskian quantities, I used a $Q^{2}$ analyticity approach, whereby each type of power correction is related to the time-like discontinuity of the corresponding term in the associated Euclidean quantity.

I further showed that, under the assumption of a universal coupling, a simple phenomenology of eventual "unconventional" $1 / Q^{2}$ power corrections can be developped, 
focussing on their channel-dependence (independently of their physical origin). The evidence for the existence of such terms is presently scarce: they have only been detected in a lattice calculation [40] of the gluon condensate. A physical picture for their occurence have also been developped in [28].

An important issue which deserves further investigation is that of renormalization scheme dependence, since the magnitude of the exprimentally extracted power corrections depend on the choice of RS. I have emphasized that the approach of $[5,4]$, if viewed as the first (single gluon exchange) term in a yet hypothetical "dressed skeleton expansion" of QCD inherits the essential built-in RS independence of the latter, since the (universal) running coupling $\bar{\alpha}_{s}\left(k^{2}\right)$ which dresses the virtual gluon propagator is supposed to be uniquely identified. Furthermore, the potentially dangerous integration over the (eventual) Landau singularity at low $k^{2}$ is avoided in the approach of [5] through the introduction of the IR cut-off $\mu_{I}$, and transmutted into the set of non-perturbative parameters which characterize the low momentum behavior of the coupling. The "infrared regularized characteristic function", combined with the "RS invariant Borel transform" are the essential tools to perform the corresponding RS invariant analysis for Minkowskian quantities.

One might worry about the convergence of the assumed "dressed skeleton expansion", especially if $\bar{\alpha}_{s}\left(k^{2}\right)$ turns out to be not particularly small in the infrared region. However, the IR magnitude of $\bar{\alpha}_{s}\left(k^{2}\right)$ may actually be irrelevant, since the infrared contribution from diagrams with two (dressed) soft gluon exchanges (I adopt a simple QED analogy for the sake of the argument) is expected to be anyway power suppressed, whatever the IR magnitude of $\bar{\alpha}_{s}\left(k^{2}\right)$, compare to that of the single (dressed) soft gluon exchange contribution (barring eventual problems related to the occurence of the "Milan factor" [45] in not inclusive enough Minkowskian quantities). Some practical questions also remain concerning the optimal choice of the IR cut-off $\mu_{I}$, which appears as an effective additionnal fit parameter in the approach of [5].

Finally, this approach relies on the assumption that the universal QCD coupling $\bar{\alpha}_{s}$ is well defined at the non-perturbative level; one might then worry whether its perturbative component $\bar{\alpha}_{s}^{P T}$ is also well defined, and is not itself affected by the IR renormalon ambiguity present in Green's functions. If this turns out to be the case, the present framework would become theoretically more difficult to justify (even if phenomenologically successful).

\section{Acknowledgments}

I have much benefited from discussions with M. Beneke, especially concerning the contents of section 5 . 


\section{A. Power corrections in Euclidean quantities}

Given $D\left(Q^{2}\right)$ in eq.(2.1), let us derive the power corrections contained in:

$$
\delta D\left(Q^{2}\right) \equiv \delta D\left(\Lambda^{2} / Q^{2}\right)=\int_{0}^{\infty} \frac{d k^{2}}{k^{2}} \delta \bar{\alpha}_{s}\left(\Lambda^{2} / k^{2}\right) \Phi_{D}\left(k^{2} / Q^{2}\right)
$$

I assume, for $k^{2} \ll Q^{2}$, the analytic (if $n$ is integer) behavior:

$$
\Phi_{D}\left(k^{2} / Q^{2}\right) \simeq A\left(\frac{k^{2}}{Q^{2}}\right)^{n}+\mathcal{O}\left[\left(\frac{k^{2}}{Q^{2}}\right)^{n+1}\right]
$$

(this case allows for a leading power correction of $U V$ origin in the associated (section 5 ) Minkowskian quantity $R$; extension of the present method to deal with logarithmic terms in eq.(A.2) is straightforward). On the other hand, for $k^{2} \gg \Lambda^{2} \mathrm{I}$ assume:

$$
\delta \bar{\alpha}_{s}\left(k^{2}\right) \equiv \delta \bar{\alpha}_{s}\left(\Lambda^{2} / k^{2}\right) \simeq\left(b+\frac{c}{\log \frac{k^{2}}{\Lambda^{2}}}\right)\left(\frac{\Lambda^{2}}{k^{2}}\right)^{n}+\mathcal{O}\left[\left(\frac{\Lambda^{2}}{k^{2}}\right)^{n+1}\right]
$$

with the same power $n$ (this is the most tricky case, and involves no real loss of generality; the case of inequal powers is dealt with below). If one then tries to use the expansions eq.(A.2) or (A.3) under the integral in eq.(A.1), one encounters UV or IR divergencies. To circumvent this problem, it is appropriate to proceed in a completely symmetrical way with respect the two relevant scales $\Lambda^{2}$ (the "small" scale) and $Q^{2}$ (the "large" scale), as well as to the two functions $\Phi_{D}$ and $\delta \bar{\alpha}_{s}$, and split the integral in eq.(A.1), at $\Lambda^{2}$ (or, more generally, at $\mu_{I}^{2} \geq \Lambda^{2}$, to deal with the Landau singularity) and $Q^{2}$ :

$$
\begin{aligned}
\delta D\left(Q^{2}\right) & =\int_{0}^{\mu_{I}^{2}} \frac{d k^{2}}{k^{2}} \delta \bar{\alpha}_{s}\left(\Lambda^{2} / k^{2}\right) \Phi_{D}\left(k^{2} / Q^{2}\right)+\int_{\mu_{I}^{2}}^{Q^{2}} \frac{d k^{2}}{k^{2}} \delta \bar{\alpha}_{s}\left(\Lambda^{2} / k^{2}\right) \Phi_{D}\left(k^{2} / Q^{2}\right) \\
& +\int_{Q^{2}}^{\infty} \frac{d k^{2}}{k^{2}} \delta \bar{\alpha}_{s}\left(\Lambda^{2} / k^{2}\right) \Phi_{D}\left(k^{2} / Q^{2}\right) \\
& \equiv \delta D_{I R}\left(Q^{2}\right)+\delta D_{U V,(-)}\left(Q^{2}\right)+\delta D_{U V,(+)}\left(Q^{2}\right)
\end{aligned}
$$

The low energy integral $\delta D_{I R}\left(Q^{2}\right)$ is conveniently merged with the corresponding integral over the perturbative part of the coupling to yield the term $D_{I R}\left(Q^{2}\right)$ in eq.(2.6) (the "infrared" power corrections). At large $Q^{2}$ one gets, using eq.(A.2):

$$
D_{I R}\left(Q^{2}\right) \simeq A K\left(\mu_{I}^{2}\right)\left(\frac{\Lambda^{2}}{Q^{2}}\right)^{n}
$$

(representing the contribution of a dimension $n$ operator in the OPE), where:

$$
K\left(\mu_{I}^{2}\right) \equiv \int_{0}^{\mu_{I}^{2}} \frac{d k^{2}}{k^{2}} \bar{\alpha}_{s}\left(k^{2}\right)\left(\frac{k^{2}}{\Lambda^{2}}\right)^{n}
$$


is a low energy moment of the "physical" coupling.

On the other hand, using eq.(A.3), one gets for the high energy integral at large $Q^{2}$ :

$$
\delta D_{U V,(+)}\left(Q^{2}\right) \simeq\left(\frac{\Lambda^{2}}{Q^{2}}\right)^{n} B\left[b+\frac{c}{\log \frac{Q^{2}}{\Lambda^{2}}}+\mathcal{O}\left(\frac{1}{\log ^{2} \frac{Q^{2}}{\Lambda^{2}}}\right)\right]
$$

where:

$$
B \equiv \int_{Q^{2}}^{\infty} \frac{d k^{2}}{k^{2}}\left(\frac{Q^{2}}{k^{2}}\right)^{n} \Phi_{D}\left(k^{2} / Q^{2}\right)
$$

To deal with the "intermediate range" integral $\delta D_{U V,(-)}\left(Q^{2}\right)$, one uses simultaneously the expansions eq.(A.2) and (A.3), and proceeds by iteration. Defining:

$$
\Phi_{D}\left(k^{2} / Q^{2}\right) \equiv A\left(\frac{k^{2}}{Q^{2}}\right)^{n}+\Phi_{D}^{(n+1)}\left(k^{2} / Q^{2}\right)
$$

and:

$$
\delta \bar{\alpha}_{s}\left(k^{2}\right) \equiv\left(b+\frac{c}{\log \frac{k^{2}}{\Lambda^{2}}}\right)\left(\frac{\Lambda^{2}}{k^{2}}\right)^{n}+\delta \bar{\alpha}_{s}^{(n+1)}\left(k^{2}\right)
$$

where $\Phi_{D}^{(n+1)}\left(k^{2} / Q^{2}\right)$ is $\mathcal{O}\left[\left(\frac{k^{2}}{Q^{2}}\right)^{n+1}\right]$ and $\delta \bar{\alpha}_{s}^{(n+1)}\left(k^{2}\right)$ is $\mathcal{O}\left[\left(\frac{\Lambda^{2}}{k^{2}}\right)^{n+1}\right]$, one gets:

$$
\begin{aligned}
\delta D_{U V,(-)}\left(Q^{2}\right) & =A\left(\frac{\Lambda^{2}}{Q^{2}}\right)^{n} \int_{\mu_{I}^{2}}^{Q^{2}} \frac{d k^{2}}{k^{2}}\left(b+\frac{c}{\log \frac{k^{2}}{\Lambda^{2}}}\right) \\
& +\left(\frac{\Lambda^{2}}{Q^{2}}\right)^{n} \int_{\mu_{I}^{2}}^{Q^{2}} \frac{d k^{2}}{k^{2}}\left(\frac{Q^{2}}{k^{2}}\right)^{n} \Phi_{D}^{(n+1)}\left(k^{2} / Q^{2}\right)\left(b+\frac{c}{\log \frac{k^{2}}{\Lambda^{2}}}\right) \\
& +A\left(\frac{\Lambda^{2}}{Q^{2}}\right)^{n} \int_{\mu_{I}^{2}}^{Q^{2}} \frac{d k^{2}}{k^{2}}\left(\frac{k^{2}}{\Lambda^{2}}\right)^{n} \delta \bar{\alpha}_{s}^{(n+1)}\left(k^{2}\right) \\
& +\int_{\mu_{I}^{2}}^{Q^{2}} \frac{d k^{2}}{k^{2}} \delta \bar{\alpha}_{s}^{(n+1)}\left(k^{2}\right) \Phi_{D}^{(n+1)}\left(k^{2} / Q^{2}\right)
\end{aligned}
$$

Now the last integral yields an $\mathcal{O}\left[\left(\frac{\Lambda^{2}}{Q^{2}}\right)^{n+1}\right]$ contribution and can be neglected, whereas, up to $\mathcal{O}\left(1 / Q^{2}\right)$ corrections we have :

$$
\begin{aligned}
\int_{\mu_{I}^{2}}^{Q^{2}} \frac{d k^{2}}{k^{2}}\left(\frac{Q^{2}}{k^{2}}\right)^{n} \Phi_{D}^{(n+1)}\left(k^{2} / Q^{2}\right)\left(b+\frac{c}{\log \frac{k^{2}}{\Lambda^{2}}}\right) & \simeq \int_{0}^{Q^{2}} \frac{d k^{2}}{k^{2}}\left(\frac{Q^{2}}{k^{2}}\right)^{n} \Phi_{D}^{(n+1)}\left(k^{2} / Q^{2}\right)\left(b+\frac{c}{\log \frac{k^{2}}{\Lambda^{2}}}\right) \\
& =C\left[b+\frac{c}{\log \frac{Q^{2}}{\Lambda^{2}}}+\mathcal{O}\left(\frac{1}{\log ^{2} \frac{Q^{2}}{\Lambda^{2}}}\right)\right]
\end{aligned}
$$

with:

$$
C \equiv \int_{0}^{Q^{2}} \frac{d k^{2}}{k^{2}}\left(\frac{Q^{2}}{k^{2}}\right)^{n} \Phi_{D}^{(n+1)}\left(k^{2} / Q^{2}\right)
$$


(where the integral is IR convergent). Furthermore, up to $\mathcal{O}\left(1 / Q^{2}\right)$ corrections we have:

$$
\int_{\mu_{I}^{2}}^{Q^{2}} \frac{d k^{2}}{k^{2}}\left(\frac{k^{2}}{\Lambda^{2}}\right)^{n} \delta \bar{\alpha}_{s}^{(n+1)}\left(k^{2}\right) \simeq \int_{\mu_{I}^{2}}^{\infty} \frac{d k^{2}}{k^{2}}\left(\frac{k^{2}}{\Lambda^{2}}\right)^{n} \delta \bar{\alpha}_{s}^{(n+1)}\left(k^{2}\right)=\text { const }
$$

since the integral is UV convergent. One deduces:

$\delta D_{U V,(-)}\left(Q^{2}\right) \simeq\left(\frac{\Lambda^{2}}{Q^{2}}\right)^{n}\left[A\left(b \log \frac{Q^{2}}{\Lambda^{2}}+c \log \log \frac{Q^{2}}{\Lambda^{2}}\right)+c o n s t+\frac{C c}{\log \frac{Q^{2}}{\Lambda^{2}}}+\mathcal{O}\left(\frac{1}{\log ^{2} \frac{Q^{2}}{\Lambda^{2}}}\right)\right]$

Thus, since:

$$
\delta D_{U V}\left(Q^{2}\right) \equiv \delta D_{U V,(-)}\left(Q^{2}\right)+\delta D_{U V,(+)}\left(Q^{2}\right)
$$

one ends up with:

$\delta D_{U V}\left(Q^{2}\right) \simeq\left(\frac{\Lambda^{2}}{Q^{2}}\right)^{n}\left[A\left(b \log \frac{Q^{2}}{\Lambda^{2}}+c \log \log \frac{Q^{2}}{\Lambda^{2}}\right)+\right.$ const $\left.+\frac{(B+C) c}{\log \frac{Q^{2}}{\Lambda^{2}}}+\mathcal{O}\left(\frac{1}{\log ^{2} \frac{Q^{2}}{\Lambda^{2}}}\right)\right]$

Note the log-enhanced terms arise because I assumed the unsufficiently suppressed behavior eq.(A.3) for $\delta \bar{\alpha}_{s}\left(k^{2}\right)$. Any more damped behavior (say with a $\mathcal{O}\left(1 / \log ^{2} k^{2}\right.$ ) term, see section 5) will only result in a leading constant term within the brackets in eq.(A.12).

In the general case where the low $k^{2}$ behavior of $\Phi_{D}\left(k^{2} / Q^{2}\right)$ and the high $k^{2}$ behavior of $\delta \bar{\alpha}_{s}\left(k^{2}\right)$ have different leading powers, one just expands either $\Phi_{D}\left(k^{2} / Q^{2}\right)$ (at small $k^{2}$ ) or $\delta \bar{\alpha}_{s}\left(k^{2}\right)$ (at large $k^{2}$ ) (depending which one has the smallest leading power, i.e. is less suppressed) under the integral in eq.(A.1), until one is back to the case of equal powers. In so doing, one never meets any IR or UV divergence until equal powers are reached. The resulting power terms can then be classified as entirely infrared (if $\Phi_{D}$ is expanded, the standard case) or entirely ultraviolet (if $\delta \bar{\alpha}_{s}$ is expanded, see e.g. eq.(5.21)).

A similar method allows to derive the large $Q^{2}$ (or, equivalently, the small $\mu^{2}$ ) expansions of the "characteristic functions" $\dot{\mathcal{F}}_{D}\left(\mu^{2} / Q^{2}\right)$ and $\dot{\mathcal{F}}_{R}\left(\mu^{2} / Q^{2}\right)$, starting from the dispersion relations eq.(3.14), (C.2), or (C.6) (where $\mu^{2}$ plays the role of $\left.\Lambda^{2}\right)$. Note that in the present method, non-analytic $\log \mu^{2}$ terms in the "gluon mass" $\mu^{2}$ arise from the "UV" part of the dispersive integrals. This fact may cause some confusion, since these terms are usually viewed [46] as the result of $I R$ divergences. This paradox is clarified by the observation that in the present derivation, one chooses $\mu_{I}^{2} \simeq \mu^{2}$, whereas the standard statement is correct if one takes $\mu^{2} \ll \mu_{I}^{2}$. This second view point was used in section 3 , where the absence of non-analytic terms at small $\mu^{2}$ 
in $\dot{\mathcal{F}}_{D, U V}\left(\mu^{2}, Q^{2}\right)$ and $\dot{\mathcal{F}}_{R, U V}\left(\mu^{2}, Q^{2}\right)$ was pointed out, despite the presence of $\log Q^{2}$ terms in their large $Q^{2}$ behavior: these functions are the analogues of $\delta D_{U V}$, and to derive their large $Q^{2}$ behavior one treats $\mu_{I}^{2}$ as an $\mathcal{O}\left(\mu^{2}\right)$ quantity. Note also the nonanalytic terms may be alternatively viewed as the result of (gluon mass insensitive) $U V$ divergences, arising e.g. from taking the expansion eq.(A.2) inside the integral in eq.(3.16).

\section{B. Power corrections in Minkowskian quantities}

When one tries to derive power corrections starting from the "Minkowskian" representation eq.(2.10), setting:

$$
\left\{\begin{array}{c}
\bar{\rho}_{s}=\bar{\rho}_{s}^{P T}+\delta \bar{\rho}_{s} \\
\bar{\alpha}_{e f f}=\bar{\alpha}_{e f f}^{P T}+\delta \bar{\alpha}_{e f f}
\end{array}\right.
$$

one has to take into account the fact that:

$$
R_{A P T}\left(Q^{2}\right) \equiv \int_{0}^{\infty} \frac{d \mu^{2}}{\mu^{2}} \bar{\alpha}_{e f f}^{P T}\left(\mu^{2}\right) \dot{\mathcal{F}}_{R}\left(\mu^{2} / Q^{2}\right)=R_{P T}\left(Q^{2}\right)+\delta R_{A P T}\left(Q^{2}\right)
$$

differs from the Borel sum $R_{P T}$ by power terms $\delta R_{A P T}$. They occur because the "Minkowskian" coupling $\bar{\alpha}_{e f f}^{P T}\left(\mu^{2}\right)$, although an entirely perturbative construct, reaches a non-trivial IR fixed point at low $\mu^{2}$ (at the difference of $\bar{\alpha}_{s}^{P T}$ ). Similarly, if one defines:

$$
\begin{aligned}
\bar{\alpha}_{s}^{A P T}\left(k^{2}\right) & =-\int_{0}^{\infty} \frac{d \mu^{2}}{\mu^{2}+k^{2}} \bar{\rho}_{s}^{P T}\left(\mu^{2}\right) \\
& =k^{2} \int_{0}^{\infty} \frac{d \mu^{2}}{\left(\mu^{2}+k^{2}\right)^{2}} \bar{\alpha}_{e f f}^{P T}\left(\mu^{2}\right)
\end{aligned}
$$

one finds that $\bar{\alpha}_{s}^{A P T}$ differs from $\bar{\alpha}_{s}^{P T}$ by power terms $\delta \bar{\alpha}_{s}^{A P T}$ :

$$
\bar{\alpha}_{s}^{A P T}\left(k^{2}\right)=\bar{\alpha}_{s}^{P T}\left(k^{2}\right)+\delta \bar{\alpha}_{s}^{A P T}\left(k^{2}\right)
$$

which remove the Landau singularity assumed to be present in $\bar{\alpha}_{s}^{P T}$. For instance, in the one-loop case where $\bar{\alpha}_{s}^{P T}\left(k^{2}\right)=1 / \beta_{0} \log \left(k^{2} / \Lambda^{2}\right)$, one obtains $\bar{\alpha}_{s}^{A P T}$ by just removing the pole, i.e.:

$$
\bar{\alpha}_{s}^{A P T}\left(k^{2}\right)=\frac{1}{\beta_{0} \ln \left(k^{2} / \Lambda^{2}\right)}-\frac{1}{\beta_{0}} \frac{1}{\frac{k^{2}}{\Lambda^{2}}-1}
$$

In general, one gets, at large $k^{2}$ :

$$
\delta \bar{\alpha}_{s}^{A P T}\left(k^{2}\right)=\sum_{n=1}^{\infty}(-1)^{n+1} b_{n}^{A P T}\left(\frac{\Lambda^{2}}{k^{2}}\right)^{n}
$$


where the constants $b_{n}^{A P T}$ cannot be easily calculated for a general perturbative coupling, since they depend on all orders [3] of the perturbative beta-function (see eq.(B.21) below). $\bar{\alpha}_{s}^{A P T}$ is the "analytic" coupling of [24], whose time-like discontinuity coincides ${ }^{18}$ with that of the perturbative coupling. It is therefore necessary to split $\bar{\alpha}_{s}$ into two pieces, each of which satisfies the dispersion relation eq.(2.13) (at the difference of the pieces in eq.(2.2)):

$$
\bar{\alpha}_{s}\left(k^{2}\right)=\bar{\alpha}_{s}^{A P T}\left(k^{2}\right)+\delta \bar{\alpha}_{s}^{A N P}\left(k^{2}\right)
$$

with:

$$
\begin{aligned}
\delta \bar{\alpha}_{s}^{A N P}\left(k^{2}\right) & =-\int_{0}^{\infty} \frac{d \mu^{2}}{\mu^{2}+k^{2}} \delta \bar{\rho}_{s}\left(\mu^{2}\right) \\
& =k^{2} \int_{0}^{\infty} \frac{d \mu^{2}}{\left(\mu^{2}+k^{2}\right)^{2}} \delta \bar{\alpha}_{e f f}\left(\mu^{2}\right)
\end{aligned}
$$

and:

$$
\delta \bar{\alpha}_{s}\left(k^{2}\right)=\delta \bar{\alpha}_{s}^{A P T}\left(k^{2}\right)+\delta \bar{\alpha}_{s}^{A N P}\left(k^{2}\right)
$$

$\left(\delta \bar{\alpha}_{s}\right.$ itself does not satisfies the dispersion relation eq.(B.8)). To deal with $\delta \bar{\alpha}_{s}^{A N P}\left(k^{2}\right)$, I make the simplifying assumption that $\delta \bar{\alpha}_{e f f}\left(\mu^{2}\right)$ is exponentially small at large $\mu^{2}$. Then, expanding the kernel under the integral in eq.(B.8) yields:

$$
\delta \bar{\alpha}_{s}^{A N P}\left(k^{2}\right)=\sum_{n=1}^{\infty}(-1)^{n+1} b_{n}^{A N P}\left(\frac{\Lambda^{2}}{k^{2}}\right)^{n}
$$

where:

$$
b_{n}^{A N P}=\int_{0}^{\infty} n \frac{d \mu^{2}}{\mu^{2}}\left(\frac{\mu^{2}}{\Lambda^{2}}\right)^{n} \delta \bar{\alpha}_{e f f}\left(\mu^{2}\right)
$$

are integer moments of $\delta \bar{\alpha}_{e f f}$. It follows that:

$$
\delta \bar{\alpha}_{s}\left(k^{2}\right)=\sum_{n=1}^{\infty}(-1)^{n+1} b_{n}\left(\frac{\Lambda^{2}}{k^{2}}\right)^{n}
$$

with

$$
b_{n}=b_{n}^{A P T}+b_{n}^{A N P}
$$

In general, both $\delta \bar{\alpha}_{s}^{A P T}\left(k^{2}\right)$ and $\delta \bar{\alpha}_{s}^{A N P}\left(k^{2}\right)$ are $\mathcal{O}\left(1 / k^{2}\right)$ at large $k^{2}$, but the total coupling modification $\delta \bar{\alpha}_{s}\left(k^{2}\right)$ itself may decrease faster, if one arranges the first few (or even all!) coefficients $b_{n}^{A P T}$ and $b_{n}^{A N P}$ to cancell each other.

\footnotetext{
${ }^{18}$ In this respect a somewhat artificial element enters the construction of $\bar{\alpha}_{s}^{A P T}$ : beyond one loop, the discontinuity of the (renormalization group improved) perturbative coupling usually starts in the space-like region. It is therefore arbitrary to trunkate this discontinuity at $\mu^{2}=0$ to enforce a causal coupling: one could just as well construct an APT coupling whose time-like discontinuity starts at $\mu^{2}=c \Lambda^{2}>0$.
} 
The proof proceeds by considering separately the contributions $R_{A P T}$ and $\delta R_{A N P}$ of $\bar{\alpha}_{e f f}^{P T}$ and $\delta \bar{\alpha}_{e f f}$ respectively to:

$$
R=R_{A P T}+\delta R_{A N P}
$$

with:

$$
\delta R=\delta R_{A P T}+\delta R_{A N P}
$$

i) Consider first $R_{A P T}\left(Q^{2}\right)$ (eq.(B.2)). To determine the power terms in $\delta R_{A P T}$ (for an Euclidean quantity, they would arise directly from the contribution of $\delta \bar{\alpha}_{s}^{A P T}$ to eq.(2.1)), one splits the integral in eq.(B.2) at $\mu^{2}=\Lambda^{2}$ :

$$
\begin{aligned}
R_{A P T}\left(Q^{2}\right) & =\int_{0}^{\Lambda^{2}} \frac{d \mu^{2}}{\mu^{2}} \bar{\alpha}_{e f f}^{P T}\left(\mu^{2}\right) \dot{\mathcal{F}}_{R}\left(\mu^{2} / Q^{2}\right)+\int_{\Lambda^{2}}^{\infty} \frac{d \mu^{2}}{\mu^{2}} \bar{\alpha}_{e f f}^{P T}\left(\mu^{2}\right) \dot{\mathcal{F}}_{R}\left(\mu^{2} / Q^{2}\right) \\
& \equiv R_{<}^{A P T}\left(Q^{2}\right)+R_{>}^{A P T}\left(Q^{2}\right)
\end{aligned}
$$

Using the Borel representation of $\bar{\alpha}_{e f f}^{P T}$ (eq.(3.2)), $R_{>}^{A P T}$ can be written as:

$$
R_{>}^{A P T}\left(Q^{2}\right)=R_{P T}\left(Q^{2}\right)-R_{<}^{P T}\left(Q^{2}\right) \equiv R_{>}^{P T}\left(Q^{2}\right)
$$

where $R_{<}^{P T}\left(Q^{2}\right)$ is the Borel sum corresponding to $R_{<}^{A P T}\left(Q^{2}\right)$ (this is the piece of $R_{P T}$ which contains the IR renormalons):

$$
R_{<}^{P T}\left(Q^{2}\right) \equiv \int_{0}^{\infty} d z \tilde{\alpha}_{e f f}(z)\left[\int_{0}^{\Lambda^{2}} \frac{d \mu^{2}}{\mu^{2}} \dot{\mathcal{F}}_{R}\left(\mu^{2} / Q^{2}\right) \exp \left(-z \beta_{0} \ln \frac{\mu^{2}}{\Lambda^{2}}\right)\right]
$$

Thus:

$$
\delta R_{A P T}\left(Q^{2}\right)=R_{<}^{A P T}\left(Q^{2}\right)-R_{<}^{P T}\left(Q^{2}\right)
$$

The power corrections contained in eq.(B.19) are obtained by expanding $\dot{\mathcal{F}}_{R}\left(\mu^{2} / Q^{2}\right)$ at large $Q^{2}$ (i.e. low $\mu^{2}$ ) inside the corresponding integrals of finite support $\left[0, \Lambda^{2}\right]$ in eq.(B.16) and (B.18) (note that, for $Q^{2}>\Lambda^{2}, \delta R_{A P T}\left(Q^{2}\right)$ depends only on the "low gluon mass piece" $\mathcal{F}_{R,(-)}$ of $\mathcal{F}_{R}$ ). For instance, an analytic term $n\left(\frac{\mu^{2}}{Q^{2}}\right)^{n}$ (with $n>0$ integer) in the low- $\mu^{2}$ expansion of $\dot{\mathcal{F}}_{R}\left(\mu^{2} / Q^{2}\right)$ contributes a power correction:

$$
b_{n}^{A P T}\left(\frac{\Lambda^{2}}{Q^{2}}\right)^{n}
$$

with:

$$
b_{n}^{A P T}=I_{n}-I_{n}^{P T}
$$

where:

$$
I_{n} \equiv \int_{0}^{\Lambda^{2}} n \frac{d \mu^{2}}{\mu^{2}}\left(\frac{\mu^{2}}{\Lambda^{2}}\right)^{n} \bar{\alpha}_{e f f}^{P T}\left(\mu^{2}\right)
$$


and:

$$
\begin{aligned}
I_{n}^{P T} & \equiv \int_{0}^{\infty} d z \tilde{\alpha}_{e f f}(z)\left[\int_{0}^{\Lambda^{2}} n \frac{d \mu^{2}}{\mu^{2}}\left(\frac{\mu^{2}}{\Lambda^{2}}\right)^{n} \exp \left(-z \beta_{0} \ln \frac{\mu^{2}}{\Lambda^{2}}\right)\right] \\
& =\int_{0}^{\infty} d z \tilde{\alpha}_{e f f}(z) \frac{1}{1-\frac{z}{z_{n}}}
\end{aligned}
$$

is the Borel sum corresponding to $I_{n}$. Applying a similar method to the kernel of the dispersion relation eq.(B.3) to derive the power corrections in $\delta \bar{\alpha}_{s}^{A P T}$ (eq.(B.6)) shows that the $b_{n}^{A P T}$ 's in eq.(B.6) and (B.21) are actually the same, which is one way to recover the results for UV power corrections of section 5 (at least those that arise from the APT part of the coupling).

Similarly, a non-analytic term $n\left(\frac{\mu^{2}}{Q^{2}}\right)^{n}\left(c_{n} \ln \frac{Q^{2}}{\mu^{2}}+d_{n}\right)$ in $\dot{\mathcal{F}}_{R}(n$ integer) contributes a log-enhanced power correction:

$$
c_{n}\left(\frac{\Lambda^{2}}{Q^{2}}\right)^{n}\left(b_{n}^{A P T} \ln \frac{Q^{2}}{\Lambda^{2}}+\bar{b}_{n}^{A P T}\right)+d_{n} b_{n}^{A P T}\left(\frac{\Lambda^{2}}{Q^{2}}\right)^{n}
$$

with:

$$
\bar{b}_{n}^{A P T}=\bar{I}_{n}-\bar{I}_{n}^{P T}
$$

where:

$$
\bar{I}_{n} \equiv \int_{0}^{\Lambda^{2}} n \frac{d \mu^{2}}{\mu^{2}}\left(\frac{\mu^{2}}{\Lambda^{2}}\right)^{n} \ln \frac{\Lambda^{2}}{\mu^{2}} \bar{\alpha}_{e f f}^{P T}\left(\mu^{2}\right)
$$

and:

$$
\begin{aligned}
\bar{I}_{n}^{P T} & \equiv \int_{0}^{\infty} d z \tilde{\alpha}_{e f f}(z)\left[\int_{0}^{\Lambda^{2}} n \frac{d \mu^{2}}{\mu^{2}}\left(\frac{\mu^{2}}{\Lambda^{2}}\right)^{n} \ln \frac{\Lambda^{2}}{\mu^{2}} \exp \left(-z \beta_{0} \ln \frac{\mu^{2}}{\Lambda^{2}}\right)\right] \\
& =\frac{1}{n} \int_{0}^{\infty} d z \tilde{\alpha}_{e f f}(z) \frac{1}{\left(1-\frac{z}{z_{n}}\right)^{2}}
\end{aligned}
$$

is the Borel sum corresponding to $\bar{I}_{n}$. Note that $\bar{I}_{n}^{P T}$, hence $\bar{b}_{n}^{A P T}$, are ambiguous, due to the presence of an IR renormalon (a simple pole) at $z=z_{n}$ in the Borel transform, the simple zero in $\tilde{\alpha}_{e f f}(z)$ only partially cancelling the double pole in the integrand of eq.(B.27). This is an example of the relation $[46,15]$ between non-analytic terms in the characteristic function and IR renormalons. This relation can only be understood if $\bar{\alpha}_{e f f}^{P T}\left(\mu^{2}\right)$ has a non-trivial IR fixed point: otherwise, if one assumes e.g. $\bar{\alpha}_{e f f}^{P T}\left(\mu^{2}\right)$ is given by the one-loop coupling (i.e. $\tilde{\alpha}_{e f f}(z) \equiv 1$ ), one could associate IR renormalons even to analytic terms in the low $\mu^{2}$ expansion of $\dot{\mathcal{F}}_{R}\left(\mu^{2} / Q^{2}\right)$ !

On the other hand, the coefficients $b_{n}^{A P T}$ of the leading-log parts (and in particular of the analytic parts if there are no accompanying log) are unambiguous for $n$ integer (eq.(B.23)), which suggests they should be associated to short-distances: this interpretation is confirmed by the results of section 5 and Appendix A. Note that all power corrections (both of UV and IR origin) formally arise (see eq.(B.19) and 
the remark below) from integration over low $\mu^{2}$, and shows it is cumbersome to use the "Minkowskian" representation eq.(2.10) to separate long from short distances, at the difference of the "Euclidean" representation eq.(2.1). In particular, $R_{>}^{A P T}\left(Q^{2}\right)$ in eq.(B.16) usually contains "unorthodox", OPE unrelated UV power contributions (the exception is the case $n \neq$ integer), which makes it unconvenient as a definition of "regularized" perturbation theory. It differs from the "OPE consistent" definition $R_{U V}^{P T}\left(Q^{2}\right)$ since it is the low gluon mass piece (below $\Lambda^{2}$ ) of $\dot{\mathcal{F}}_{R}$, rather then that of its discontinuity, which is removed. It may also be misleading, since the "unorthodox" contributions could be removed by similar contributions in the sum $R_{<}^{A P T}\left(Q^{2}\right)+\delta R_{A N P}\left(Q^{2}\right)$ (see below).

ii) Consider next the contribution of $\delta \bar{\alpha}_{e f f}$ :

$$
\delta R_{A N P}\left(Q^{2}\right) \equiv \int_{0}^{\infty} \frac{d \mu^{2}}{\mu^{2}} \delta \bar{\alpha}_{e f f}\left(\mu^{2}\right) \dot{\mathcal{F}}_{R}\left(\mu^{2} / Q^{2}\right)
$$

If one makes again the simplifying assumption that $\delta \bar{\alpha}_{e f f}\left(\mu^{2}\right)$ is exponentially suppressed at large $\mu^{2}$, the corresponding power corrections are obtained by taking the low $\mu^{2}$ expansion of $\dot{\mathcal{F}}_{R}\left(\mu^{2} / Q^{2}\right)$ inside the integral in eq.(B.28). For instance, a nonanalytic term $n\left(\frac{\mu^{2}}{Q^{2}}\right)^{n}\left(c_{n} \ln \frac{Q^{2}}{\mu^{2}}+d_{n}\right)$ in $\dot{\mathcal{F}}_{R}(n$ integer $)$ contributes a log-enhanced power correction:

$$
c_{n}\left(\frac{\Lambda^{2}}{Q^{2}}\right)^{n}\left(b_{n}^{A N P} \ln \frac{Q^{2}}{\Lambda^{2}}+\bar{b}_{n}^{A N P}\right)+d_{n} b_{n}^{A N P}\left(\frac{\Lambda^{2}}{Q^{2}}\right)^{n}
$$

where $b_{n}^{A N P}$ is given in eq.(B.11), and:

$$
\bar{b}_{n}^{A N P}=\int_{0}^{\infty} n \frac{d \mu^{2}}{\mu^{2}}\left(\frac{\mu^{2}}{\Lambda^{2}}\right)^{n} \ln \frac{\Lambda^{2}}{\mu^{2}} \delta \bar{\alpha}_{e f f}\left(\mu^{2}\right)
$$

Note that eq.(B.29) has exactly the same structure as the corresponding contribution to $\delta R_{A P T}\left(Q^{2}\right)$ (eq.(B.24)) with the substitutions $b_{n}^{A P T} \rightarrow b_{n}^{A N P}$ and $\bar{b}_{n}^{A P T} \rightarrow \bar{b}_{n}^{A N P \text { ! }}$ Again, the leading log terms terms with a coefficient $b_{n}^{A N P}$ (an analytic, integer moment) should be associated to short distances, while the sub-leading log terms, with a coefficient $\bar{b}_{n}^{A N P}$ (a non-analytic moment), are partly long distance.

Application to the causal perturbative coupling: this is the case where $\bar{\alpha}_{s}^{P T} \equiv \bar{\alpha}_{s}^{A P T}$, and $\delta \bar{\alpha}_{s}^{A P T} \equiv 0$. Then the $b_{n}^{A P T}$ 's must all vanish, which implies that the power corrections in $R_{A P T}\left(Q^{2}\right)$ are "OPE compatible", since only non-analytic terms in $\dot{\mathcal{F}}_{R}$ can contribute.

Furthermore, for a general $\bar{\alpha}_{s}^{P T}$ (not necessarily causal) one can show that power corrections in $R_{U V}^{A P T}\left(Q^{2}\right)$ involve only the $b_{n}^{A P T}$ 's, and therefore all vanish when specialized to a causal coupling. To prove the former statement, I note that a large $Q^{2}$ contribution to $\dot{\mathcal{F}}_{R, U V}\left(\mu^{2}, Q^{2}\right)$ of the form $n\left(\frac{\mu^{2}}{Q^{2}}\right)^{n}\left(c_{n} \ln \frac{Q^{2}}{\Lambda^{2}}+d_{n}\left(\mu^{2} / \Lambda^{2}\right)\right)$ (I take for simplicity the IR cut-off $\mu_{I}$ equal to $\Lambda$ ) contributes a power correction:

$$
\left(\frac{\Lambda^{2}}{Q^{2}}\right)^{n}\left(c_{n} b_{n}^{A P T} \ln \frac{Q^{2}}{\Lambda^{2}}+J_{n}-J_{n}^{P T}\right)
$$


where:

$$
J_{n} \equiv \int_{0}^{\Lambda^{2}} n \frac{d \mu^{2}}{\mu^{2}}\left(\frac{\mu^{2}}{\Lambda^{2}}\right)^{n} d_{n}\left(\frac{\mu^{2}}{\Lambda^{2}}\right) \bar{\alpha}_{e f f}^{P T}\left(\mu^{2}\right)
$$

and $J_{n}^{P T}$ is the corresponding Borel sum. But $d_{n}\left(\mu^{2} / \Lambda^{2}\right)$ is analytic at small $\mu^{2}$ (see the comments at the end of Appendix A), and consequently, expanding $d_{n}\left(\mu^{2} / \Lambda^{2}\right)$ in powers of $\mu^{2}$ under the integral in eq.(B.32) and its analogue in $J_{n}^{P T}$ (it is easy to check that the radius of convergence of the series is $\mu^{2} / \Lambda^{2}=1$ ) yields only terms proportionnal to the integer moments $b_{p}^{A P T}$.

\section{Expressing Minkowskian power corrections in term of $\delta \bar{\alpha}_{s}$}

Let us give a proof of eq.(2.16) alternative to that of [3]. The proof proceeds again by considering separately the contributions $R_{A P T}$ and $\delta R_{A N P}$ of $\bar{\alpha}_{e f f}^{P T}$ and $\delta \bar{\alpha}_{e f f}$.

i) Consider first $R_{A P T}\left(Q^{2}\right)$ (eq.(B.2)). An explicit expression for $\delta R_{A P T}$ has been obtained in the one-loop case in [15]. The following can be seen as an extension of their result. Splitting the integral in eq.(B.2) at $Q^{2}$, it is clear the piece above $Q^{2}$ contributes only to the Borel sum. It is therefore sufficient to consider:

$$
R_{(-)}^{A P T}\left(Q^{2}\right) \equiv \int_{0}^{Q^{2}} \frac{d \mu^{2}}{\mu^{2}} \bar{\alpha}_{e f f}^{P T}\left(\mu^{2}\right) \dot{\mathcal{F}}_{R,(-)}\left(\mu^{2} / Q^{2}\right)
$$

which shares the same power terms as $R_{A P T}$. The expression for $\delta R_{A P T}$ depends on the form of the dispersion relation satisfied by $\mathcal{F}_{R,(-)}$.

Assume for simplicity one subtraction at $\mu^{2}=0$. Then:

$$
\dot{\mathcal{F}}_{R,(-)}\left(\mu^{2} / Q^{2}\right)=\mu^{2} \int_{0}^{\infty} \frac{d k^{2}}{\left(k^{2}+\mu^{2}\right)^{2}} \Phi_{R}\left(k^{2} / Q^{2}\right)
$$

Substituting eq.(C.2) into eq.(C.1) yields:

$$
R_{(-)}^{A P T}\left(Q^{2}\right)=\int_{0}^{\infty} \frac{d k^{2}}{k^{2}} \bar{\alpha}_{s,(-)}^{A P T}\left(k^{2}, Q^{2}\right) \Phi_{R}\left(k^{2} / Q^{2}\right)
$$

with:

$$
\bar{\alpha}_{s,(-)}^{A P T}\left(k^{2}, Q^{2}\right) \equiv k^{2} \int_{0}^{Q^{2}} \frac{d \mu^{2}}{\left(\mu^{2}+k^{2}\right)^{2}} \bar{\alpha}_{e f f}^{P T}\left(\mu^{2}\right)
$$

It is clear however that the power terms in $\bar{\alpha}_{s,(-)}^{A P T}$ are the same as those in $\bar{\alpha}_{s}^{A P T}$, since again the integration range above $Q^{2}$ in the dispersion relation eq.(B.3) contributes only to the Borel sum. Thus:

$$
\bar{\alpha}_{s,(-)}^{A P T}\left(k^{2}, Q^{2}\right)=\bar{\alpha}_{s,(-)}^{P T}\left(k^{2}, Q^{2}\right)+\delta \bar{\alpha}_{s}^{A P T}\left(k^{2}\right)
$$

(where $\bar{\alpha}_{s,(-)}^{P T}$ is defined as a Borel sum) and:

$$
R_{(-)}^{A P T}\left(Q^{2}\right)=R_{(-)}^{P T}\left(Q^{2}\right)+\delta R_{A P T}\left(Q^{2}\right)
$$


with:

$$
R_{(-)}^{P T}\left(Q^{2}\right)=\int_{0}^{\infty} \frac{d k^{2}}{k^{2}} \bar{\alpha}_{s,(-)}^{P T}\left(k^{2}, Q^{2}\right) \Phi_{R}\left(k^{2} / Q^{2}\right)
$$

and:

$$
\delta R_{A P T}\left(Q^{2}\right)=\int_{0}^{\infty} \frac{d k^{2}}{k^{2}} \delta \bar{\alpha}_{s}^{A P T}\left(k^{2}\right) \Phi_{R}\left(k^{2} / Q^{2}\right)
$$

Assume next two subtractions at $\mu^{2}=0$. Then:

$$
\dot{\mathcal{F}}_{R,(-)}\left(\mu^{2} / Q^{2}\right)=a_{0} \frac{\mu^{2}}{Q^{2}}+\mu^{2} \int_{0}^{\infty} d k^{2}\left[\frac{1}{\left(k^{2}+\mu^{2}\right)^{2}}-\frac{1}{k^{4}}\right] \Phi_{R}\left(k^{2} / Q^{2}\right)
$$

where $a_{0}$ is a subtraction constant, and $\Phi_{R}\left(k^{2} / Q^{2}\right)$ is assumed to be $\mathcal{O}\left(k^{4} / Q^{4}\right)$ at small $k^{2}$, in order to have no IR divergence in the dispersive integral. Substituting eq.(C.6) into eq.(C.1) yields:

$$
R_{(-)}^{A P T}\left(Q^{2}\right)=a_{0} R_{1}^{A P T}\left(Q^{2}\right)+\int_{0}^{\infty} \frac{d k^{2}}{k^{2}}\left[\bar{\alpha}_{s,(-)}^{A P T}\left(k^{2}, Q^{2}\right)-\frac{Q^{2}}{k^{2}} R_{1}^{A P T}\left(Q^{2}\right)\right] \Phi_{R}\left(k^{2} / Q^{2}\right)
$$

where:

$$
R_{1}^{A P T}\left(Q^{2}\right) \equiv \int_{0}^{Q^{2}} \frac{d \mu^{2}}{\mu^{2}} \frac{\mu^{2}}{Q^{2}} \bar{\alpha}_{e f f}^{P T}\left(\mu^{2}\right)
$$

Again, $R_{1}^{A P T}\left(Q^{2}\right)$ differs from its Borel sum $R_{1}^{P T}\left(Q^{2}\right)$ by a power correction $b_{1}^{A P T} \frac{\Lambda^{2}}{Q^{2}}$, and one can easily show [3] with the method of Appendix B that $b_{1}^{A P T}$ is the same coefficient which appears in the leading term in eq.(B.6). The power corrections in eq.(C.7) are therefore obtained by substituting $R_{1}^{A P T}$ and $\bar{\alpha}_{s,(-)}^{A P T}$ by they respective power corrections pieces $b_{1}^{A P T} \frac{\Lambda^{2}}{Q^{2}}$ and $\delta \bar{\alpha}_{s}^{A P T}\left(k^{2}\right)$, which yields:

$$
\delta R_{A P T}\left(Q^{2}\right)=a_{0} b_{1}^{A P T} \frac{\Lambda^{2}}{Q^{2}}+\int_{0}^{\infty} \frac{d k^{2}}{k^{2}}\left[\delta \bar{\alpha}_{s}^{A P T}\left(k^{2}\right)-b_{1}^{A P T} \frac{\Lambda^{2}}{k^{2}}\right] \Phi_{R}\left(k^{2} / Q^{2}\right)
$$

where the integrand is properly subtracted to insure convergence at large $k^{2}$ (similar expressions may be obtained if the subtractions are performed away from $\mu^{2}=0$ ).

ii) Consider next $\delta R_{A N P}\left(Q^{2}\right)$ (eq.(B.28)). If one makes again the simplifying assumption that $\delta \bar{\alpha}_{e f f}\left(\mu^{2}\right)$ is exponentially suppressed at large $\mu^{2}$, one gets:

$$
\delta R_{A N P}\left(Q^{2}\right) \simeq \int_{0}^{\infty} \frac{d \mu^{2}}{\mu^{2}} \delta \bar{\alpha}_{e f f}\left(\mu^{2}\right) \dot{\mathcal{F}}_{R,(-)}\left(\mu^{2} / Q^{2}\right)
$$

up to exponentially small corrections at large $Q^{2}$. It is then straightforward to express the right-hand side of eq.(C.10) in term of $\delta \bar{\alpha}_{s}^{A N P}$. Assuming for instance the dispersion relation eq.(C.6), and using the dispersion relation eq.(B.8), one gets:

$$
\delta R_{A N P}\left(Q^{2}\right) \simeq a_{0} b_{1}^{A N P} \frac{\Lambda^{2}}{Q^{2}}+\int_{0}^{\infty} \frac{d k^{2}}{k^{2}}\left[\delta \bar{\alpha}_{s}^{A N P}\left(k^{2}\right)-b_{1}^{A N P} \frac{\Lambda^{2}}{k^{2}}\right] \Phi_{R}\left(k^{2} / Q^{2}\right)
$$


with $b_{1}^{A N P}$ given in eq.(B.11). Eq.(C.11) has the same form as eq.(C.9). Adding the two yields the final result for $\delta R$ :

$$
\delta R\left(Q^{2}\right) \simeq a_{0} b_{1} \frac{\Lambda^{2}}{Q^{2}}+\int_{0}^{\infty} \frac{d k^{2}}{k^{2}}\left[\delta \bar{\alpha}_{s}\left(k^{2}\right)-b_{1} \frac{\Lambda^{2}}{k^{2}}\right] \Phi_{R}\left(k^{2} / Q^{2}\right)
$$

where $b_{1}=b_{1}^{A P T}+b_{1}^{A N P}$, which is independent of the split in eq.(B.7), and is correct at large $Q^{2}$ up to exponentially small corrections within the present assumptions. If one further assumes that $\delta \bar{\alpha}_{s}\left(k^{2}\right)$ decreases faster then $1 / k^{2}$, then $b_{1}=0$, and one recovers eq.(2.16) (faster decrease may be necessary to make the right hand side of eq.(2.16) ultraviolet convergent, depending on the number of assumed subtractions in the dispersion relation for $\left.\mathcal{F}_{R,(-)}\right)$. Note also the results of this section are also valid in the more general case where $\mathcal{F}_{R,(-)}\left(\mu^{2} / Q^{2}\right)$ can be written as the sum of a function which satisfies a dispersion relation (hence has no complex singularities) and a function analytic around the origin - a generalized "subtraction term" (but which may have complex singularities at finite distance from the origin, i.e. for large enough $\left.\mu^{2} / Q^{2}\right)$. 


\section{References}

[1] V.I. Zakharov, in Proceedings of YKIS97, Kyoto, December 1997 [hep-ph/9802416].

[2] M. Beneke, [hep-ph/9807443].

[3] G. Grunberg, [hep-ph/9705290].

[4] Yu.L. Dokshitzer, G. Marchesini and B.R. Webber, Nucl. Phys. B469 (1996) 93.

[5] Yu.L. Dokshitzer and B.R. Webber, Phys.Lett. B352 (1995) 451.

[6] Yu.L. Dokshitzer, V.A. Khoze and S.I. Troyan, Phys.Rev. D53 (1996) 89.

[7] Yu.L. Dokshitzer, A. Lucenti, G. Marchesini and G.P. Salam, JHEP 05 (1998) 003.

[8] M. Dasgupta, G.E. Smye and B.R. Webber, JHEP 04 (1998) 017.

[9] E. Gardi, G. Grunberg and M. Karliner, JHEP 07 (1998) 007.

[10] G. Grunberg, in Proceedings of the International Europhysics Conference on HighEnergy Physics (HEP 97), Jerusalem, Israel, 1997 [hep-ph/9711481].

[11] S.J. Brodsky, G.P. Lepage and P.B. Mackenzie, Phys.Rev. D28 (1983) 228; G.P. Lepage and P.B. Mackenzie, Phys.Rev. D48 (1993) 2250.

[12] H.J. Lu and C.A.R. Sa de Melo, Phys.Lett. B273 (1991) 260 (Erratum: ibid. B285 (1992) 399); H.J.Lu, Phys.Rev. D45 (1992) 1217; SLAC-0406 (Ph.D. Thesis) (1992).

[13] M. Neubert, Phys.Rev. D51 (1995) 5924; [hep-ph/9502264].

[14] D.J. Broadhurst and A.G. Grozin, Phys.Rev. D52 (1995) 4082.

[15] M. Beneke and V.M. Braun, Phys.Lett. B348 (1995) 513; P. Ball, M. Beneke and V.M. Braun, Nucl. Phys. B452 (1995) 563.

[16] N.J. Watson Nucl. Phys. B494 (1997) 388.

[17] S. Catani, G. Marchesini and B.R. Webber, Nucl. Phys. B349 (1991) 635.

[18] V.A. Novikov, M.A. Shifman, A.I. Vainshtein and V.I. Zakharov, Phys.Rep. 116 (1984) 104; Nucl. Phys. B249 (1985) 445.

[19] A.H. Mueller, Nucl.Phys. B250 (1985) 327.

[20] V.I. Zakharov, Nucl.Phys. B385 (1992) 452.

[21] G. Grunberg, Phys.Lett. B304 (1993) 183; in Quantum Field Theoretic Aspects of High Energy Physics, Kyffhauser, Germany, September 1993.

[22] M. Beneke, Nucl.Phys. B405 (1993) 424. 
[23] L.S. Brown and L.G. Yaffe, Phys.Rev. D45 (1992) 398; L.S. Brown, L.G. Yaffe and C. Zhai, Phys.Rev. D46 (1992) 4712.

[24] I.L. Solovtsov and D.V. Shirkov, Phys.Rev.Lett. 79 (1997) 1209.

[25] G. Grunberg, Phys.Lett. B372 (1996) 121.

[26] Yu.L. Dokshitzer and N.G. Uraltsev, Phys.Lett. B380 (1996) 141.

[27] P. Ball, M. Beneke and V.M. Braun Phys.Rev. D52 (1995) 3929.

[28] R. Akhoury and V.I. Zakharov, in Proceedings of the 5th International Conference on Physics Beyond the Standard Model, Balholm, Norway, May 1997 [hep-ph/9705318]; Nucl.Phys. 64 (Proc. Suppl.) (1998) 350; Phys.Lett. B438 (1998) 165.

[29] M. Beneke, V.M. Braun and N. Kivel, [hep-ph/9809287].

[30] M. Beneke, private communication.

[31] S. Peris and E. de Rafael, Nucl.Phys. B500 (1997) 325.

[32] K.G. Chetyrkin, S. Narison and V.I. Zakharov, [hep-ph/9811275].

[33] S. Narison, Phys.Lett. B361 (1995) 121.

[34] G. Grunberg, Phys.Rev. D29 (1984) 2315.

[35] G. Grunberg, Phys.Lett. B349 (1995) 469.

[36] M. Beneke, Nucl.Phys. B405 (1993) 424.

[37] V.N. Gribov (private communication from Yu.L. Dokshitzer).

[38] Yu.L. Dokshitzer, [hep-ph/9812252].

[39] B.R. Webber, JHEP 10 (1998) 012.

[40] G. Burgio, F. Di Renzo, G. Marchesini and E. Onofri, Phys.Lett. B422 (1998) 219; Nucl. Phys. 63 Proc. Suppl. (1998) 805.

[41] A.I. Alekseev, [hep-ph/9802372]; A.I Alekseev and B.A. Arbuzov, Mod.Phys.Lett. A13 (1998) 1747.

[42] J.M. Campbell, E.W.N. Glover and C.J. Maxwell, Phys.Rev.Lett. 81 (1998) 1568.

[43] M. Beneke and V.M. Braun, Nucl.Phys. 51C (Proc. Suppl.) (1996) 217.

[44] G. Grunberg, in preparation.

[45] Yu.L. Dokshitzer, A. Lucenti, G. Marchesini and G.P. Salam, Nucl.Phys. B511 (1998) 396.

[46] M. Beneke, V.M. Braun and V.I. Zakharov, Phys.Rev.Lett. 73 (1994) 3058. 